\title{
OPTIMAL DECAY OF EXTREMALS FOR THE FRACTIONAL SOBOLEV INEQUALITY
}

\author{
LORENZO BRASCO, SUNRA MOSCONI, AND MARCO SQUASSINA
}

\begin{abstract}
We obtain the sharp asymptotic behavior at infinity of extremal functions for the fractional critical Sobolev embedding.
\end{abstract}

\section{Contents}

1. Introduction and main result 1

2. Preliminary results 5

2.1. Notation 5

2.2. Elementary inequalities 5

2.3. Functional framework 6

2.4. Radial functions 10

3. Properties of extremals 11

3.1. Basic properties 11

3.2. Decay estimates 15

4. Proof of the main result 19

Appendix A. Power functions 25

References 30

\section{INTRODUCTION AND MAIN RESUlt}

Let $N>p>1$. In two seminal papers, T. Aubin [3] and G. Talenti [33] showed that the minimizers of the Sobolev quotient

$$
\mathcal{S}_{p}=\inf _{u \in D^{1, p}\left(\mathbb{R}^{N}\right) \backslash\{0\}} \frac{\|\nabla u\|_{L^{p}\left(\mathbb{R}^{N}\right)}^{p}}{\left(\int_{\mathbb{R}^{N}}|u|^{\frac{N p}{N-p}} d x\right)^{\frac{N-p}{N}}},
$$

are given by the family of functions

$$
U_{t}(x)=\mathcal{C} t^{\frac{p-N}{p}} U\left(\frac{x-x_{0}}{t}\right), \quad \mathcal{C} \in \mathbb{R} \backslash\{0\}, t>0, x_{0} \in \mathbb{R}^{N},
$$

where

$$
U(x)=\mathcal{C}_{N, p}\left(1+|x|^{\frac{p}{p-1}}\right)^{\frac{p-N}{p}}, \quad \mathcal{C}_{N, p}=\left(\int_{\mathbb{R}^{N}}\left(1+|x|^{\frac{p}{p-1}}\right)^{-N} d x\right)^{\frac{N p}{p-N}} .
$$

For the limit case $p=1$, the problem was investigated by H. Federer and W. H. Fleming in [14] and by V. G. Mazya in [27].

2010 Mathematics Subject Classification. 46E35, 35B40, 49K22.

Key words and phrases. Fractional $p$-Laplacian, critical Sobolev embedding, extremals, decay. 
On one side, these results establish an enlightening connection between the theory of Sobolev spaces and the theory of classical isoperimetric inequalities. On the other side, they provide a very powerful tool for the study of second order partial differential equations involving nonlinearities reaching the critical growth with respect to the Sobolev embedding. In the case $p=2$, these classification results were formally derived by G. Rosen in [30].

The variational problem (1.1) is related to the following equation involving the $p$-Laplace operator $\Delta_{p} u=\operatorname{div}\left(|\nabla u|^{p-2} \nabla u\right)$,

$$
-\Delta_{p} u=|u|^{\frac{N p}{N-p}-2} u, \quad \text { in } \mathbb{R}^{N} .
$$

In fact, a nontrivial problem is that of proving that the only fixed sign solutions of this equation are precisely given by (1.2), for a suitable choice of the constant.

In the restricted class of radially symmetric fixed sign solutions to (1.3), this was shown by M. Guedda and L. Veron in [17]. Recently, in [34, Corollary 1.3] for the case $1<p \leq$ $2 N /(N+2)$, in [10, Theorem 1.2] for the case $2 N /(N+2)<p \leq 2$ and in [32, Theorem 1.1] for the case $2<p<N$, it was proved that any positive weak solution to (1.3) is radially symmetric and radially decreasing about some point, thus answering positively to the classification of constant sign solutions to (1.3).

The result by Aubin and Talenti, as well as the previous results in the linear case $p=2$, strongly rely on the reduction of the problem to an ordinary differential equation which can be explicitly solved. We recall that more recently, the Aubin-Talenti result has been reproved in [8, Theorem 2] by means of very different techniques, based on Optimal Transport.

Let now $s \in(0,1), p>1$ and $N>s p$. The goal of this paper is to provide information about the asymptotic behavior at infinity of optimizers of the problem

$$
\mathcal{S}_{p, s}:=\inf _{u \in D^{s, p}\left(\mathbb{R}^{N}\right) \backslash\{0\}} \frac{\int_{\mathbb{R}^{2 N}} \frac{|u(x)-u(y)|^{p}}{|x-y|^{N+s p}} d x d y}{\left(\int_{\mathbb{R}^{N}}|u|^{\frac{N p}{N-s p}} d x\right)^{\frac{N-s p}{N}}},
$$

which is related to the fractional Sobolev embedding, see for example [28, Theorem 1]. Here

$$
D^{s, p}\left(\mathbb{R}^{N}\right)=\left\{u \in L^{N p /(N-s p)}\left(\mathbb{R}^{N}\right): \int_{\mathbb{R}^{2 N}} \frac{|u(x)-u(y)|^{p}}{|x-y|^{N+s p}} d x d y<\infty\right\} .
$$

In the limit case $p=1$, the sharp constant above has been determined in [15, Theorem 4.1] (see also [6, Theorem 4.10]). The relevant extremals are given by characteristic functions of balls, exactly as in the local case.

Problem (1.4) for $p>1$ is now related to the study of the nonlocal integro-differential equation

$$
\left(-\Delta_{p}\right)^{s} u=|u|^{\frac{N p}{N-s p}-2} u, \quad \text { in } \mathbb{R}^{N},
$$

where, formally, the operator $\left(-\Delta_{p}\right)^{s}$ is defined on smooth functions as

$$
\left(-\Delta_{p}\right)^{s} u(x)=2 \lim _{\varepsilon \searrow 0} \int_{\mathbb{R}^{N} \backslash B_{\varepsilon}(x)} \frac{|u(x)-u(y)|^{p-2}(u(x)-u(y))}{|x-y|^{N+s p}} d y, \quad x \in \mathbb{R}^{N} .
$$

This operator appears in some recent works like [2] and [21]. See also [11,18-20,22] and the references therein for some existence and regularity results. 
In the Hilbertian case $p=2$, it is known by [9, Theorem 1.1] that the family of functions

$$
U_{t}(x)=\mathcal{C} t^{\frac{2 s-N}{2}}\left(1+\left(\frac{\left|x-x_{0}\right|}{t}\right)^{2}\right)^{\frac{2 s-N}{2}}, \quad \mathcal{C} \in \mathbb{R} \backslash\{0\}, t>0, x_{0} \in \mathbb{R}^{N},
$$

is the only set of minimizers for the best Sobolev constant $\mathcal{S}_{2, s}$. More precisely, in $[9$, Theorem 1.1] it is proved that the family (1.6) provides all the minimizers of the following problem

$$
\widetilde{\mathcal{S}}_{2, s}:=\inf _{u \in D^{s, 2}\left(\mathbb{R}^{N}\right) \backslash\{0\}} \frac{\left\|(-\Delta)^{s / 2} u\right\|_{L^{2}\left(\mathbb{R}^{N}\right)}^{2}}{\left(\int_{\mathbb{R}^{N}}|u|^{\frac{2 N}{N-2 s}} d x\right)^{\frac{N-2 s}{N}}},
$$

where the $L^{2}$ norm of $(-\Delta)^{s / 2} u$ is defined in terms of the Fourier transform. By using [12, Proposition 3.6], one knows that

$$
\int_{\mathbb{R}^{2 N}} \frac{|u(x)-u(y)|^{2}}{|x-y|^{N+2 s}} d x d y=c\left\|(-\Delta)^{s / 2} u\right\|_{L^{2}\left(\mathbb{R}^{N}\right)}^{2},
$$

for some $c=c(N, s)>0$. This implies that (1.6) are the only solutions of (1.4) as well.

It is also known by [7, Theorem 1] that, for a suitable positive constant $\mathcal{C}=\mathcal{C}(N, s),(1.6)$ are the only positive solutions of

$$
(-\Delta)^{s} u=|u|^{\frac{2 N}{N-2 s}-2} u \quad \text { in } \mathbb{R}^{N} .
$$

The result in [7] is based upon the full equivalence between the weak solutions to (1.7) and the integral formulation

$$
u(x)=\int_{\mathbb{R}^{N}} \frac{|u(y)|^{\frac{2 N}{N-2 s}-2} u}{|x-y|^{N-2 s}} d y, \quad u \in L^{\frac{2 N}{N-2 s}}\left(\mathbb{R}^{N}\right),
$$

on the validity of some Kelvin transform and on moving plane arguments applied to (1.8), in the spirit of [23].

Unfortunately, in the nonlocal and nonlinear case $p \neq 2$ there is no Kelvin transform and no equivalent integral representation result. Furthermore, even restricting to the class of radially symmetric functions, establishing a classification result for the optimizers of (1.4) seems very hard. We conjecture that the optimizers are given by

$$
U_{t}(x)=\mathcal{C} t^{\frac{s p-N}{p}} U\left(\frac{x-x_{0}}{t}\right), \quad \mathcal{C} \in \mathbb{R} \backslash\{0\}, t>0, x_{0} \in \mathbb{R}^{N},
$$

where this time

$$
U(x):=\mathcal{C}_{N, p, s}\left(1+|x|^{\frac{p}{p-1}}\right)^{\frac{s p-N}{p}}, \quad \mathcal{C}_{N, p, s}=\left(\int_{\mathbb{R}^{N}}\left(1+|x|^{\frac{p}{p-1}}\right)^{-N} d x\right)^{\frac{N p}{s p-N}}
$$

Notice that (1.9) and (1.10) are consistent with the cases $p=2$ or $s=1$, in the last case we are back to the family of Aubin-Talenti functions (1.2) for the $p$-Laplacian operator.

In the main result of this paper, we prove that extremals for (1.4) have exactly the decay rate at infinity dictated by formula (1.10). Namely, we have the following. 
Theorem 1.1. Let $U \in D^{s, p}\left(\mathbb{R}^{N}\right)$ be any minimizer for (1.4). Then $U \in L^{\infty}\left(\mathbb{R}^{N}\right)$ is a constant sign, radially symmetric and monotone function with

$$
\lim _{|x| \rightarrow \infty}|x|^{\frac{N-s p}{p-1}} U(x)=U_{\infty}
$$

for some constant $U_{\infty} \in \mathbb{R} \backslash\{0\}$.

Remark 1.2. As it will be apparent from the proof of Theorem 1.1, the same conclusion (1.11) can be drawn for any constant sign, radially symmetric and monotone solution of the critical equation (1.5). In the local case this property is a plain consequence of the aforementioned classification result of [17].

The building blocks of Theorem 1.1 are a weak $L^{q}$ estimate for the minimizers (Proposition 3.3), a Radial Lemma for Lorentz spaces (Lemma 2.9) and the fact that the function

$$
\Gamma(x):=|x|^{-\frac{N-s p}{p-1}}, \quad x \in \mathbb{R}^{N} \backslash\{0\},
$$

is a weak solution of $\left(-\Delta_{p}\right)^{s} u=0$ in $\mathbb{R}^{N} \backslash B_{r}$, for any $r>0$ (Theorem A.4). Then the crucial point will be constructing suitable barrier functions to be combined with a version of the comparison principle for $\left(-\Delta_{p}\right)^{s}$ recently obtained in [19]. Observe that for $s=1$, the function $\Gamma$ above is nothing but the fundamental solution of the $p$-Laplacian.

We wish to stress that Theorem 1.1 also provides a very useful tool for the investigation of existence of weak solutions for the nonlocal Brezis-Nirenberg problem in a smooth bounded domain $\Omega \subset \mathbb{R}^{N}$, i.e.

$$
\begin{cases}\left(-\Delta_{p}\right)^{s} u=\lambda|u|^{p-2} u+|u|^{\frac{N p}{N-s p}-2} u & \text { in } \Omega, \\ u=0 & \text { in } \mathbb{R}^{N} \backslash \Omega,\end{cases}
$$

where $\lambda>0$. This problem has been studied in [31] for $p=2$. For a general exponent $1<p<N / s$, by means of (1.11), one can estimate truncations of $U_{t}$ via a suitable cutoff function in terms of the sharp constant $\mathcal{S}_{p, s}$ without knowing the explicit form of the optimizers. Such a procedure is new even for the local case. These estimates allow to apply mountain pass or linking arguments by forcing the min-max levels to fall inside a compactness range for the energy functionals, see [29] for more details.

Plan of the paper. In Section 2 we set all the notations, definitions and basic facts that will be needed throughout the paper. Then in Section 3 we prove existence of solutions for (1.4), together with some basic integrability properties. We also prove that extremals have to be comparable to

$$
x \mapsto|x|^{-\frac{N-s p}{p-1}}, \quad x \in \mathbb{R}^{N} \backslash\{0\},
$$

at infinity (Corollary 3.7). Then the exact behavior (1.11) is proved in Section 4. The paper ends with Appendix A, containing a rigourous computation of the fractional $p$-Laplacian of a power function.

Acknowledgements. We warmly thank Yannick Sire for some informal discussions on the subject of this paper. We owe Remark 1.2 to the kind courtesy of an anonymous referee, we wish to thank him. This research has been partially supported by Gruppo Nazionale per l'Analisi Matematica, la Probabilità e le loro Applicazioni (INdAM) and by Agence Nationale de la Recherche, through the project ANR-12-BS01-0014-01 GeOMETRYA. Part of this paper 
was written during a visit of S.M. and M. S. in Marseille in March 2015. The I2M and FRUMAM institutions are gratefully acknowledged.

\section{Preliminary Results}

2.1. Notation. In the following we will fix $s \in(0,1), p>1$ and $N$ as the dimension, letting for brevity

$$
p^{*}=\frac{N p}{N-s p} .
$$

We denote by $\omega_{N}$ the measure of the $N$-dimensional ball having unit radius. Moreover, $\mathbf{S}^{N-1}$ will denote $\left\{x \in \mathbb{R}^{N}:|x|=1\right\}$. For $E \subseteq \mathbb{R}^{N}$ measurable we denote by $|E|$ its $N$-dimensional Lebesgue measure, by $E^{c}=\mathbb{R}^{N} \backslash E$ its complement and by $\chi_{E}$ its characteristic function. If $u: E \rightarrow \mathbb{R}$ is measurable we set

$$
[u]_{W^{s, p}(E)}^{p}:=\int_{E \times E} \frac{|u(x)-u(y)|^{p}}{|x-y|^{N+s p}} d x d y, \quad[u]_{s, p}:=[u]_{W^{s, p}\left(\mathbb{R}^{N}\right)},
$$

and for any $q>0$

$$
\|u\|_{L^{q}(E)}:=\left(\int_{E}|u|^{q} d x\right)^{1 / q}, \quad\|u\|_{q}:=\|u\|_{L^{q}\left(\mathbb{R}^{N}\right)} .
$$

Finally, for $t \in \mathbb{R}$ we will use the notation

$$
J_{p}(t)=|t|^{p-2} t
$$

2.2. Elementary inequalities. We list here some useful inequalities on the function $J_{p}$. First, consider the case $p \geq 2$. We recall that

$$
\left|J_{p}(a)-J_{p}(b)\right| \leq(p-1)\left(|a|^{p-2}+|b|^{p-2}\right)|a-b|, \quad a, b \in \mathbb{R}, \quad p \geq 2,
$$

as a consequence of the mean value Theorem. In [19, eq. (2.7)] it is also proved the following inequality

$$
J_{p}(a)-J_{p}(a+b) \leq-2^{2-p} b^{p-1}, \quad a \in \mathbb{R}, b \geq 0, \quad p \geq 2 .
$$

Let us consider the case $p \in(1,2]$. We recall the well-known monotonicity inequality

$$
\left(J_{p}(a)-J_{p}(b)\right)(a-b) \geq c \frac{|a-b|^{2}}{\left(a^{2}+b^{2}\right)^{\frac{2-p}{2}}}, \quad a, b \in \mathbb{R} \backslash\{0\}, \quad p \in(1,2] .
$$

Next we prove the following inequality

$$
J_{p}(a)-J_{p}(a-b) \geq \max \left\{J_{p}(A)-J_{p}(A-b),\left(\frac{b}{2}\right)^{p-1}\right\}, \quad a \in[0, A], b \geq 0, p \in(1,2] .
$$

We distinguish two cases. First suppose that $a \geq b / 2$. The function $t \mapsto J_{p}(t)-J_{p}(t-b)$ is readily seen to be decreasing on $[b / 2,+\infty[$, so that

$$
J_{p}(a)-J_{p}(a-b) \geq J_{p}(A)-J_{p}(A-b)
$$

in this case. On the other hand, if $a<b / 2$, being $J_{p}$ odd and increasing we have

$$
J_{p}(a)-J_{p}(a-b) \geq J_{p}(b-a) \geq J_{p}\left(\frac{b}{2}\right),
$$

and thus (2.4) is proved. 
2.3. Functional framework. We consider the space

$$
D_{0}^{s, p}(\Omega):=\left\{u \in L^{p^{*}}(\Omega): u \equiv 0 \text { in } \Omega^{c},[u]_{s, p}<+\infty\right\}, \quad D^{s, p}\left(\mathbb{R}^{N}\right):=D_{0}^{s, p}\left(\mathbb{R}^{N}\right),
$$

which is a Banach space with respect to the norm $[\cdot]_{s, p}$. Our first aim is to prove, under suitable regularity assumptions on $\partial \Omega$, that $C_{c}^{\infty}(\Omega)$ is dense in $D_{0}^{s, p}(\Omega)$ with respect to the norm $[\cdot]_{s, p}$. While this density result is well-known for $D_{0}^{s, p}(\Omega) \cap L^{p}(\Omega)$ (see for example [13]), we will need to remove the $L^{p}$ assumption in the following. Finally we will prove a comparison principle in a rather general space.

Theorem 2.1. Let $\Omega \subset \mathbb{R}^{N}$ be an open set such that $\partial \Omega$ is compact and locally the graph of a continuous function. Then $D_{0}^{s, p}(\Omega)$ is the completion of $C_{c}^{\infty}(\Omega)$ with respect to the norm $[\cdot]_{s, p}$.

Proof. Let $u \in D_{0}^{s, p}(\Omega)$. Reasoning on $u_{+}$and $u_{-}$separately (which still belong to $D_{0}^{s, p}(\Omega)$ ), we can suppose that $u$ is nonnegative. Consider, for $\varepsilon>0$, the function $u_{\varepsilon}=(u-\varepsilon)_{+}$. Using the 1 -Lipschitzianity of $t \mapsto(t-\varepsilon)_{+}$it is readily checked that

$$
\left|u_{\varepsilon}(x)-u_{\varepsilon}(y)\right|^{p} \leq|u(x)-u(y)|^{p}, \quad\left|u_{\varepsilon}(x)-u_{\varepsilon}(y)\right|^{p} \rightarrow|u(x)-u(y)|^{p} \text {, a.e. in } \mathbb{R}^{2 N} .
$$

Therefore $u_{\varepsilon} \in D_{0}^{s, p}(\Omega)$ and by dominated convergence $\left[u_{\varepsilon}\right]_{s, p} \rightarrow[u]_{s, p}$. This in turn implies that $u_{\varepsilon} \rightarrow u$ in $D_{0}^{s, p}(\Omega)$ by uniform convexity of the norm. Now Chebyshev's inequality ensures that $\operatorname{supp}\left(u_{\varepsilon}\right)$ has finite measure, thus by Hölder's inequality we get $u_{\varepsilon} \in L^{p}\left(\mathbb{R}^{N}\right)$. This yields

$$
u_{\varepsilon} \in D_{0}^{s, p}(\Omega) \cap L^{p}\left(\mathbb{R}^{N}\right),
$$

and [13, Theorem 6$]$ ensures that $u_{\varepsilon}$ can be approximated, in the norm $[\cdot]_{s, p}$, by functions which belong to $C_{c}^{\infty}(\Omega)$.

We recall the following nonlocal Hardy inequality proved in [15, Theorem 2].

Proposition 2.2 (Hardy's inequality). Let $N>s p$. Then there exists $C=C(N, p, s)>0$ such that

$$
\int_{\mathbb{R}^{N}} \frac{|u|^{p}}{|x|^{s p}} d x \leq C[u]_{s, p}^{p}, \quad \text { for every } u \in D^{s, p}\left(\mathbb{R}^{N}\right) .
$$

We then define a suitable space where a comparison principle holds true. For any $\Omega \subset \mathbb{R}^{N}$ open set, we define

$$
\begin{gathered}
\widetilde{D}^{s, p}(\Omega):=\left\{u \in L_{\text {loc }}^{p-1}\left(\mathbb{R}^{N}\right) \cap L^{p^{*}}(\Omega): \exists E \supset \Omega \text { with } E^{c} \text { compact, } \operatorname{dist}\left(E^{c}, \Omega\right)>0\right. \\
\text { and } \left.[u]_{W^{s, p}(E)}<+\infty\right\} .
\end{gathered}
$$

We wish to point out that the definition above is given having in mind the case of $\Omega$ being an exterior domain, i.e. the complement of a compact set. Essentially, we consider functions $u$ which are regular in a slight enlargement of $\Omega$ and possibly rough far from $\Omega$.

The following expedient result will be used in the sequel.

Lemma 2.3 (Nash-type interpolation inequality). Let $1<p<\infty$ and $0<s<1$. For every $u \in L^{p-1}\left(B_{R}\right)$ such that $[u]_{W^{s, p}\left(B_{R}\right)}<+\infty$ we have

$$
\|u\|_{L^{p}\left(B_{R}\right)}^{p} \leq C R^{s p}[u]_{W^{s, p}\left(B_{R}\right)}^{p}+\frac{C}{R^{\frac{N}{p-1}}}\|u\|_{L^{p-1}\left(B_{R}\right)}^{p},
$$


for some $C=C(N, s, p)>0$.

Proof. We observe that it is enough to prove (2.6) for $R=1$, then the general case can be obtained with a simple scaling argument.

At first, we prove (2.6) for functions in $W^{s, p}\left(B_{1}\right)$. We can use a standard compactness argument: assume by contradiction that $(2.6)$ is false on $W^{s, p}\left(B_{1}\right)$, then there exists a sequence $\left\{u_{n}\right\}_{n \in \mathbb{N}} \subset W^{s, p}\left(B_{1}\right)$ such that

$$
\left\|u_{n}\right\|_{L^{p}\left(B_{1}\right)}^{p}=1 \quad \text { and } \quad\left[u_{n}\right]_{W^{s, p}\left(B_{R}\right)}^{p}+\left\|u_{n}\right\|_{L^{p-1}\left(B_{1}\right)}^{p} \leq \frac{1}{n} .
$$

In particular, the sequence is bounded in $W^{s, p}\left(B_{1}\right)$. Thus by compactness of the embedding $W^{s, p}\left(B_{1}\right) \hookrightarrow L^{p}\left(B_{1}\right)$ (see for example [12, Theorem 7.1]) we get that (up to a subsequence) it converges strongly in $L^{p}\left(B_{1}\right)$ to $u \in W^{s, p}\left(B_{1}\right)$. From (2.7) we now easily get a contradiction. This shows that (2.6) is true for functions in $W^{s, p}\left(B_{1}\right)$.

We now take $u \in L^{p-1}\left(B_{1}\right)$ with finite Gagliardo seminorm. Observe that

$$
|| u(x)|-| u(y)|| \leq|u(x)-u(y)|,
$$

so that

$$
[|u|]_{W^{s, p}\left(B_{1}\right)} \leq[u]_{W^{s, p}\left(B_{1}\right)}
$$

Thus we can assume $u$ to be positive without loss of generality. We define the increasing sequence $u_{n}=\min \{u, n\} \in W^{s, p}\left(B_{1}\right)$. From the first part of the proof and 1-Lipschitzianity of the function $t \mapsto \min \{t, n\}$ we have

$$
\begin{aligned}
\left\|u_{n}\right\|_{L^{p}\left(B_{1}\right)}^{p} & \leq C\left[u_{n}\right]_{W^{s, p}\left(B_{1}\right)}^{p}+C\left\|u_{n}\right\|_{L^{p-1}\left(B_{1}\right)}^{p} \\
& \leq C[u]_{W^{s, p}\left(B_{1}\right)}^{p}+C\left\|u_{n}\right\|_{L^{p-1}\left(B_{1}\right)}^{p} .
\end{aligned}
$$

Passing to the limit and using the Monotone Convergence we get the desired conclusion.

Lemma 2.4. Let $1<p<\infty$ and $0<s<1$. For every $u \in L_{\text {loc }}^{p-1}\left(\mathbb{R}^{N}\right)$, every $E \subset \mathbb{R}^{N}$ open set and every ball $B_{R} \subset E$, we have

$$
\int_{E} \frac{|u(x)|^{p}}{(1+|x|)^{N+s p}} d x \leq C[u]_{W^{s, p}(E)}^{p}+C\|u\|_{L^{p-1}\left(B_{R}\right)}^{p},
$$

for some $C=C(N, p, s, R)>0$, blowing-up as $R \searrow 0$.

Proof. We assume that the right-hand side on (2.9) is finite, otherwise there is nothing to prove. For simplicity, we can suppose that $B_{R}$ is centered at the origin. From (2.6), we infer

$$
\int_{B_{R}} \frac{|u|^{p}}{(1+|x|)^{N+s p}} d x \leq C R^{s p}[u]_{W^{s, p}\left(B_{R}\right)}^{p}+\frac{C}{R^{\frac{N}{p-1}}}\|u\|_{L^{p-1}\left(B_{R}\right)}^{p} .
$$

On the smaller ball $B_{R / 2}$ (still centered at the origin), we have

$$
\int_{E \backslash B_{R}} \int_{B_{R / 2}} \frac{|u(x)-u(y)|^{p}}{|x-y|^{N+s p}} d y d x \leq[u]_{W^{s, p}(E)}^{p}<+\infty .
$$

Since

$$
|x-y| \leq \frac{3}{2}|x|, \quad x \in E \backslash B_{R}, y \in B_{R / 2},
$$


we get

$$
\begin{aligned}
\int_{E \backslash B_{R}} \int_{B_{R / 2}} \frac{|u(x)-u(y)|^{p}}{|x-y|^{N+s p}} d y d x & \geq c R^{N} \int_{E \backslash B_{R}} \frac{|u|^{p}}{|x|^{N+s p}} d x \\
& -c\left(\int_{E \backslash B_{R}} \frac{1}{|x|^{N+s p}} d x\right) \int_{B_{R / 2}}|u|^{p} d y .
\end{aligned}
$$

In conclusion, the previous estimate proves

$$
\int_{E \backslash B_{R}} \frac{|u|^{p}}{(1+|x|)^{N+s p}} d x \leq \frac{C}{R^{N}}[u]_{W^{s, p}(E)}^{p}+\frac{C}{R^{N+s p}} \int_{B_{R / 2}}|u|^{p} d x .
$$

Using (2.6) to estimate the $L^{p}$ norm in the right-hand side gives

$$
\int_{E \backslash B_{R}} \frac{|u|^{p}}{(1+|x|)^{N+s p}} d x \leq \frac{C}{R^{N}}[u]_{W^{s, p}(E)}^{p}+\frac{C}{R^{N \frac{p}{p-1}+s p}}\left(\int_{B_{R / 2}}|u|^{p-1} d y\right)^{\frac{p}{p-1}},
$$

possibly for a different constant $C=C(N, s, p)>0$. By summing up (2.10) and (2.11) we get the conclusion.

The next proposition shows that in the space $\widetilde{D}^{s, p}(\Omega)$, the operator $\left(-\Delta_{p}\right)^{s}$ is well defined.

Proposition 2.5. For any $u \in \widetilde{D}^{s, p}(\Omega)$, the operator

$$
D_{0}^{s, p}(\Omega) \ni \varphi \mapsto\left\langle\left(-\Delta_{p}\right)^{s} u, \varphi\right\rangle:=\int_{\mathbb{R}^{N} \times \mathbb{R}^{N}} \frac{J_{p}(u(x)-u(y))(\varphi(x)-\varphi(y))}{|x-y|^{N+s p}} d x d y
$$

is well defined and belongs to the dual space $\left(D_{0}^{s, p}(\Omega)\right)^{*}$.

Proof. We proceed as in [19, Lemma 2.3]. Let $E \supset \Omega$ be such that $E^{c}$ is compact, $\operatorname{dist}\left(E^{c}, \Omega\right)>$ 0 and $[u]_{W^{s, p}(E)}<+\infty$. Since $\varphi \equiv 0$ in $\Omega^{c}$, we split the integral as

$$
\begin{aligned}
\int_{\mathbb{R}^{N} \times \mathbb{R}^{N}} & \frac{J_{p}(u(x)-u(y))(\varphi(x)-\varphi(y))}{|x-y|^{N+s p}} d x d y \\
& =\int_{E \times E} \frac{J_{p}(u(x)-u(y))(\varphi(x)-\varphi(y))}{|x-y|^{N+s p}}+2 \int_{\Omega \times E^{c}} \frac{J_{p}(u(x)-u(y)) \varphi(x)}{|x-y|^{N+s p}} d x d y .
\end{aligned}
$$

By Hölder's inequality the first term is finite and defines a continuous linear functional on $D_{0}^{s, p}(\Omega)$. Let us focus on the second one. By using that $\varphi \equiv 0$ in $E^{c}$, we need to show that

$$
\varphi \mapsto \int_{\Omega} \varphi(x)\left(\int_{E^{c}} \frac{J_{p}(u(x)-u(y))}{|x-y|^{N+s p}} d y\right) d x,
$$

is a continuous linear functional on $D_{0}^{s, p}(\Omega)$. By means of Hardy's inequality (2.5), we get that convergence of $\left\{\varphi_{n}\right\}_{n \in \mathbb{N}}$ in $D_{0}^{s, p}(\Omega) \subset D^{s, p}\left(\mathbb{R}^{N}\right)$ implies strong convergence in $L^{p}(\Omega)$ of $\left\{|x|^{-s} \varphi_{n}\right\}_{n \in \mathbb{N}}$. Thus to prove the claim it suffices to show that

$$
x \mapsto|x|^{s} \int_{E^{c}} \frac{J_{p}(u(x)-u(y))}{|x-y|^{N+s p}} d y \in L^{p^{\prime}}(\Omega) .
$$

Being $E^{c}$ compact and $\operatorname{dist}\left(E^{c}, \Omega\right) \geq \delta>0$ it holds

$$
|x-y| \geq C(1+|x|), \quad \text { for every } x \in \Omega, y \in E^{c},
$$


for some $C=C(E, \Omega)>0$. Thus, for almost every $x \in \Omega$, we can estimate

$$
\left|\int_{E^{c}} \frac{|x|^{s} J_{p}(u(x)-u(y))}{|x-y|^{N+s p}} d y\right| \leq C\left[\left|E^{c}\right| \frac{|u(x)|^{p-1}}{(1+|x|)^{\frac{N+s p}{p^{\prime}}}}+\frac{1}{(1+|x|)^{N+s(p-1)}} \int_{E^{c}}|u|^{p-1} d y\right] \text {. }
$$

The first term belongs to $L^{p^{\prime}}(\Omega)$ due to $(2.9)$. For the second one this follows from a direct computation. This proves the claim and the proposition.

Definition 2.6. Let $u \in \widetilde{D}^{s, p}(\Omega)$ and $\Lambda \in\left(D_{0}^{s, p}(\Omega)\right)^{*}$. We say that $\left(-\Delta_{p}\right)^{s} u \leq \Lambda$ weakly in $\Omega$ if for all $\varphi \in D_{0}^{s, p}(\Omega), \varphi \geq 0$ in $\Omega$,

$$
\int_{\mathbb{R}^{2 N}} \frac{J_{p}(u(x)-u(y))(\varphi(x)-\varphi(y))}{|x-y|^{N+s p}} d x d y \leq\langle\Lambda, \varphi\rangle .
$$

Theorem 2.7 (Comparison principle in general domains). Let $\Omega \subset \mathbb{R}^{N}$ be an open set. Let $u, v \in \widetilde{D}^{s, p}(\Omega)$ satisfy

$$
u \leq v \quad \text { in } \Omega^{c} \quad \text { and } \quad\left(-\Delta_{p}\right)^{s} u \leq\left(-\Delta_{p}\right)^{s} v \quad \text { in } \Omega .
$$

Then $u \leq v$ in $\Omega$.

Proof. It suffices to proceed as in [26, Lemma 9], we only need to prove that $w:=(u-v)_{+}$is an admissible test function, i.e. it belongs to $D_{0}^{s, p}(\Omega)$. Clearly $w \equiv 0$ in $\Omega^{c}$ and $w \in L^{p^{*}}\left(\mathbb{R}^{N}\right)$. To estimate the Gagliardo seminorm, let $E \supset \Omega$ be such that $E^{c}$ is compact, $\operatorname{dist}\left(E^{c}, \Omega\right)>0$ and

$$
\int_{E \times E} \frac{|u(x)-u(y)|^{p}}{|x-y|^{N+s p}} d x d y+\int_{E \times E} \frac{|v(x)-v(y)|^{p}}{|x-y|^{N+s p}} d x d y<+\infty .
$$

Then

$$
\int_{\mathbb{R}^{2 N}} \frac{|w(x)-w(y)|^{p}}{|x-y|^{N+s p}} d x d y=\int_{E \times E} \frac{|w(x)-w(y)|^{p}}{|x-y|^{N+s p}} d x d y+2 \int_{\Omega \times E^{c}} \frac{|w(x)|^{p}}{|x-y|^{N+s p}} d x d y,
$$

and the first integral is finite due to

$$
|w(x)-w(y)|^{p} \leq C\left(|u(x)-u(y)|^{p}+|v(x)-v(y)|^{p}\right),
$$

and (2.13). For the second one we use (2.12), and since $|w(x)|^{p} \leq C\left(|u(x)|^{p}+|v(x)|^{p}\right)$ we get

$$
\int_{\Omega \times E^{c}} \frac{|w(x)|^{p}}{|x-y|^{N+s p}} d x d y \leq C\left|E^{c}\right| \int_{\Omega} \frac{|u(x)|^{p}}{(1+|x|)^{N+s p}} d x+C\left|E^{c}\right| \int_{\Omega} \frac{|v(x)|^{p}}{(1+|x|)^{N+s p}} d x .
$$

The last two terms are finite, due the definition of $\widetilde{D}^{s, p}(\Omega)$ and $(2.9)$.

Finally, for the reader's convenience we recall the following result from [19]. The proof is identical to the one of [19, Lemma 2.8] and we omit it.

Proposition 2.8 (Non-local behavior of $\left.\left(-\Delta_{p}\right)^{s}\right)$. Let $N>s p$ and let $\Omega \subset \mathbb{R}^{N}$ be an open set such that $\partial \Omega$ is compact and locally the graph of continuous functions. Suppose that $u \in \widetilde{D}^{s, p}(\Omega)$ weakly solves $\left(-\Delta_{p}\right)^{s} u=f$ for some $f \in L_{\text {loc }}^{1}(\Omega) \cap\left(D_{0}^{s, p}(\Omega)\right)^{*}$, in the sense that

$$
\left\langle\left(-\Delta_{p}\right)^{s} u, \varphi\right\rangle=\int_{\Omega} f \varphi d x, \quad \text { for every } \varphi \in D_{0}^{s, p}(\Omega)
$$

Let $v$ be a measurable function with compact support $K:=\operatorname{supp}(v)$ such that

$$
\operatorname{dist}(K, \Omega)>0, \quad \int_{\Omega^{c}}|v|^{p-1} d x<+\infty,
$$


and define for a.e. Lebesgue point $x \in \Omega$ of $u$

$$
h(x)=2 \int_{K} \frac{J_{p}((u(x)-u(y))-v(y))-J_{p}(u(x)-u(y))}{|x-y|^{N+s p}} d y .
$$

Then $u+v \in \widetilde{D}^{s, p}(\Omega)$ and $\left(-\Delta_{p}\right)^{s}(u+v)=f+h$ weakly.

2.4. Radial functions. For every measurable function $u: \mathbb{R}^{N} \rightarrow \mathbb{R}$ we define its distribution function

$$
\mu_{u}(t)=|\{x:|u(x)|>t\}|, \quad t>0 .
$$

Let $0<q<\infty$ and $0<\theta<\infty$, the Lorentz space $L^{q, \theta}\left(\mathbb{R}^{N}\right)$ is defined by

$$
L^{q, \theta}\left(\mathbb{R}^{N}\right)=\left\{u: \int_{0}^{\infty} t^{\theta-1} \mu_{u}(t)^{\frac{\theta}{q}} d t<+\infty\right\}
$$

In the limit case $\theta=\infty$, this is defined by

$$
L^{q, \infty}\left(\mathbb{R}^{N}\right)=\left\{u: \sup _{t>0} t \mu_{u}(t)^{\frac{1}{q}}<+\infty\right\},
$$

and we recall that this coincides with the weak $L^{q}$ space (see for example [24, page 106]).

Lemma 2.9 (Radial Lemma for Lorentz spaces). Let $0<\theta \leq \infty$ and $0<q<\infty$. Let $u \in L^{q, \theta}\left(\mathbb{R}^{N}\right)$ be a non-negative and radially symmetric decreasing function. Then

$$
\begin{aligned}
& 0 \leq u(x) \leq\left(\theta \omega_{N}^{-\frac{\theta}{q}} \int_{0}^{\infty} t^{\theta-1} \mu_{u}(t)^{\frac{\theta}{q}} d t\right)^{\frac{1}{\theta}}|x|^{-\frac{N}{q}}, \quad \text { if } \theta<\infty, \\
& 0 \leq u(x) \leq\left(\omega_{N}^{-\frac{1}{q}} \sup _{t>0} t \mu_{u}(t)^{\frac{1}{q}}\right)|x|^{-\frac{N}{q}}, \quad \text { if } \theta=\infty .
\end{aligned}
$$

Proof. For simplicity, we simply write $\mu$ in place of $\mu_{u}$ and suppose that $u=u(r)$ coincides with its right-continuous representative. We start with the case $\theta<\infty$. First of all, we prove that

$$
\int_{0}^{\infty} t^{\theta-1} \mu(t)^{\frac{\theta}{q}} d t=\frac{N-\alpha}{N \theta \omega_{N}^{\alpha / N}} \int_{\mathbb{R}^{N}} \frac{u^{\theta}}{|x|^{\alpha}} d x
$$

where the exponent $\alpha<N$ is given by the relation ${ }^{1}$

With a simple change of variable

$$
\frac{\theta}{q}=\frac{N-\alpha}{N}
$$

Then we observe that

$$
\int_{\mathbb{R}^{N}} \frac{u^{\theta}}{|x|^{\alpha}} d x=\int_{\mathbb{R}^{N}} \frac{\int_{0}^{\infty} \chi_{\left\{t: u(x)^{\theta}>t\right\}}(s) d s}{|x|^{\alpha}} d x=\int_{0}^{\infty} \int_{\mathbb{R}^{N}} \frac{\chi_{\left\{t: u(x)>t^{1 / \theta}\right\}}(s)}{|x|^{\alpha}} d x d s,
$$

and

$$
\int_{0}^{\infty} t^{\theta-1} \mu(t)^{\frac{\theta}{q}} d t=\frac{1}{\theta} \int_{0}^{\infty} \mu\left(s^{1 / \theta}\right)^{\frac{\theta}{q}} d s .
$$

$$
\chi_{\left\{t: u(x)>t^{1 / \theta}\right\}}(s)=\chi_{\left\{y: u(y)>s^{1 / \theta}\right\}}(x) .
$$

\footnotetext{
${ }^{1}$ Observe that if $\theta>q$, then $\alpha<0$.
} 
By assumption we have

$$
\left\{y: u(y)>s^{1 / \theta}\right\}=\left\{y:|y|<\left(\frac{\mu\left(s^{1 / \theta}\right)}{\omega_{N}}\right)^{\frac{1}{N}}\right\}=: B_{R(s)},
$$

since the function $u$ is radially decreasing. Thus we arrive at

$$
\begin{aligned}
\int_{\mathbb{R}^{N}} \frac{u^{\theta}}{|x|^{\alpha}} d x & =\int_{0}^{\infty}\left(\int_{B_{R(s)}} \frac{1}{|x|^{\alpha}} d x\right) d s \\
& =N \omega_{N} \int_{0}^{\infty} \int_{0}^{R(s)} \varrho^{N-1-\alpha} d \varrho d s=\frac{N \omega_{N}}{N-\alpha} \int_{0}^{\infty} \frac{\mu\left(s^{1 / \theta}\right)^{\frac{N-\alpha}{N}}}{\omega_{N}^{\frac{N-\alpha}{N}}} d s \\
& =\frac{N \omega_{N}^{\alpha / N}}{N-\alpha} \int_{0}^{\infty} \mu\left(s^{1 / \theta}\right)^{\frac{\theta}{q}} d s .
\end{aligned}
$$

Using (2.16) we finally obtain

$$
\int_{0}^{\infty} t^{\theta-1} \mu(t)^{\frac{\theta}{q}} d t=\frac{N-\alpha}{N \theta \omega_{N}^{\alpha / N}} \int_{\mathbb{R}^{N}} \frac{u^{\theta}}{|x|^{\alpha}} d x
$$

which proves (2.15).

As for the decay estimate, thanks to (2.15) we have

$$
\begin{aligned}
+\infty>\int_{\mathbb{R}^{N}} \frac{u^{\theta}}{|x|^{\alpha}} d x & =N \omega_{N} \int_{0}^{+\infty} u(\varrho)^{\theta} \varrho^{N-1-\alpha} d \varrho \\
& \geq N \omega_{N} \int_{0}^{R} u(\varrho)^{\theta} \varrho^{N-1-\alpha} d \varrho \geq N \omega_{N} u(R)^{\theta} \frac{R^{N-\alpha}}{N-\alpha},
\end{aligned}
$$

where $\alpha$ is as above. Recalling that $(N-\alpha) / \theta=N / q$, we get the desired conclusion.

For the case $\theta=\infty$, it is sufficient to observe that

$$
\sup _{t>0} t \mu(t)^{\frac{1}{q}}=\omega_{N}^{\frac{1}{q}} \sup _{x \in \mathbb{R}^{N}}|x|^{\frac{N}{q}} u(x) .
$$

Then the decay estimate easily follows.

\section{Properties of extremals}

3.1. Basic properties. We first observe that by homogeneity we can equivalently write

$$
\mathcal{S}_{p, s}=\inf _{u \in D^{s, p}\left(\mathbb{R}^{N}\right)}\left\{\int_{\mathbb{R}^{2 N}} \frac{|u(x)-u(y)|^{p}}{|x-y|^{N+s p}} d x d y: \int_{\mathbb{R}^{N}}|u|^{N p /(N-s p)} d x=1\right\} .
$$

Then we start with the following result.

Proposition 3.1. Let $1<p<\infty$ and $s \in(0,1)$ be such that $s p<N$. Then:

- problem (3.1) admits a solution;

- for every $U \in D^{s, p}\left(\mathbb{R}^{N}\right)$ solving (3.1), there exist $x_{0} \in \mathbb{R}^{N}$ and $u: \mathbb{R}^{+} \rightarrow \mathbb{R}$ constant sign monotone function such that $U(x)=u\left(\left|x-x_{0}\right|\right)$; 
- every minimizer $U \in D^{s, p}\left(\mathbb{R}^{N}\right)$ weakly solves

$$
\left(-\Delta_{p}\right)^{s} U=\mathcal{S}_{p, s}|U|^{p^{*}-2} U, \quad \text { in } \mathbb{R}^{N},
$$

that is

$$
\begin{aligned}
& \qquad \int_{\mathbb{R}^{2 N}} \frac{J_{p}(U(x)-U(y))(\varphi(x)-\varphi(y))}{|x-y|^{N+s p}} d x d y=\mathcal{S}_{p, s} \int_{\mathbb{R}^{N}}|U|^{p^{*}-2} U \varphi d x, \\
& \text { for every } \varphi \in D^{s, p}\left(\mathbb{R}^{N}\right) .
\end{aligned}
$$

Proof. The existence of a solution for (3.1) follows from the Concentration-Compactness Principle, see [25, Section I.4, Example iii)].

It is not difficult to show that every solution of (3.1) must have costant sign. Indeed, for every admissible $u \in D^{s, p}\left(\mathbb{R}^{N}\right)$, still by (2.8) the function $|u|$ is still admissible and does not increase the value of the functional. More important, the inequality sign in (2.8) is strict if $u(x) u(y)<0$, i.e. if $u$ changes sign.

Radial symmetry of the solutions comes from the Pólya-Szegö principle for Gagliardo seminorms (see [1]), i.e. for every non-negative function $u \in D^{s, p}\left(\mathbb{R}^{N}\right)$ we have

$$
\left[u^{\#}\right]_{s, p}^{p} \leq[u]_{s, p}^{p} .
$$

Here $u^{\#}$ denotes the radially symmetric decreasing rearrangement of $u$. It is crucial to observe that inequality (3.3) is strict, unless $u$ is (up to a translation) a radially symmetric decreasing function, see [15, Theorem A.1].

Finally, if $U$ solves (3.1), then it minimizes as well the functional

$$
u \mapsto[u]_{s, p}^{p}-\mathcal{S}_{p, s}\left(\int_{\mathbb{R}^{N}}|u|^{p^{*}} d x\right)^{\frac{p}{p^{*}}} .
$$

Equation (3.2) is exactly the Euler-Lagrange equation associated with this functional, once it is observed that $U$ has unitary $L^{p^{*}}$ norm.

Proposition 3.2 (Global boundedness). Let $U \in D^{s, p}\left(\mathbb{R}^{N}\right)$ be a non-negative solution of (3.1). Then we have $U \in L^{\infty}\left(\mathbb{R}^{N}\right) \cap C^{0}\left(\mathbb{R}^{N}\right)$.

Proof. Thanks to the properties of the minimizers contained in Proposition 3.1, it is enough to prove that $U \in L_{\text {loc }}^{\infty}\left(\mathbb{R}^{N}\right)$, since continuity then follows from [5, Theorem 3.13] (see also [19, Theorem 5.4] for a direct proof). With this aim, we just need to show that $U \in L^{q\left(p^{*}-1\right)}\left(\mathbb{R}^{N}\right)$ for some $q>N /(s p)$. This would imply that

$$
U^{p^{*}-1} \in L^{q}\left(\mathbb{R}^{N}\right), \quad \text { for some } q>\frac{N}{s p},
$$

and thus $U \in L_{\mathrm{loc}}^{\infty}\left(\mathbb{R}^{N}\right)$ would automatically follow by [5, Theorem 3.8].

Let $M>0$ and $\alpha>1$, we set for simplicity $U_{M}=\min \{U, M\}$ and $g_{\alpha, M}(t)=t \min \{t, M\}^{\alpha-1}$. Then we insert in (3.2) the test function $\varphi=g_{\alpha, M}(U) \in D^{s, p}\left(\mathbb{R}^{N}\right)$. This yields

$$
\int_{\mathbb{R}^{2 N}} \frac{J_{p}(U(x)-U(y))\left(g_{\alpha, M}(U(x))-g_{\alpha, M}(U(y))\right)}{|x-y|^{N+s p}} d x d y=\mathcal{S}_{p, s} \int_{\mathbb{R}^{N}} U^{p^{*}-p} U_{M}^{\alpha-1} U^{p} d x .
$$

We now observe that if we set

$$
G_{\alpha, M}(t)=\int_{0}^{t} g_{\alpha, M}^{\prime}(\tau)^{\frac{1}{p}} d \tau
$$


by using [5, Lemma A.2] from the previous identity with simple manipulations we get

$$
\begin{aligned}
\int_{\mathbb{R}^{2 N}} & \frac{\left|G_{\alpha, M}(U(x))-G_{\alpha, M}(U(y))\right|^{p}}{|x-y|^{N+s p}} d x d y \\
& \leq \mathcal{S}_{p, s}\left[K_{0}^{\alpha-1} \int_{\mathbb{R}^{N}} U^{p^{*}} d x+\left(\int_{\left\{U \geq K_{0}\right\}} U^{p^{*}} d x\right)^{\frac{p^{*}-p}{p^{*}}}\left(\int_{\mathbb{R}^{N}}\left(U_{M}^{(\alpha-1)} U^{p}\right)^{\frac{p^{*}}{p}} d x\right)^{\frac{p}{p^{*}}}\right],
\end{aligned}
$$

for some $K_{0}>0$ that will be chosen in a while. If we estimate from below the left-hand side by Sobolev inequality and use that $U$ has unitary norm, we get ${ }^{2}$

$$
\begin{aligned}
\left(\frac{p}{p+\alpha-1}\right)^{p}\left(\int_{\mathbb{R}^{N}}\right. & \left.\left(U^{p} U_{M}^{(\alpha-1)}\right)^{\frac{p^{*}}{p}} d x\right)^{\frac{p}{p^{*}}} \leq K_{0}^{\alpha-1} \\
& +\left(\int_{\left\{U \geq K_{0}\right\}} U^{p^{*}} d x\right)^{\frac{p^{*}-p}{p^{*}}}\left(\int_{\mathbb{R}^{N}}\left(U_{M}^{(\alpha-1)} U^{p}\right)^{\frac{p^{*}}{p}} d x\right)^{\frac{p}{p^{*}}} .
\end{aligned}
$$

We now choose the parameters: we first take $\alpha>1$ such that

$$
p^{*}+(\alpha-1) \frac{p^{*}}{p}=q\left(p^{*}-1\right), \quad \text { i. e. } \quad \alpha=p q \frac{\left(p^{*}-1\right)}{p^{*}}-(p-1),
$$

where $q>N /(s p)$, then we choose $K_{0}=K_{0}(\alpha, U)>0$ such that

$$
\left(\int_{\left\{U \geq K_{0}\right\}} U^{p^{*}} d x\right)^{\frac{p^{*}-p}{p^{*}}} \leq \frac{1}{2}\left(\frac{p}{p+\alpha-1}\right)^{p} .
$$

With this choice we can absorb the last term on the right-hand side of (3.4) and thus obtain

$$
\left(\frac{p}{p+\alpha-1}\right)^{p}\left(\int_{\mathbb{R}^{N}} U^{p^{*}} U_{M}^{(\alpha-1) \frac{p^{*}}{p}} d x\right)^{\frac{p}{p^{*}}} \leq 2 K_{0}^{\alpha-1} .
$$

If we now take the limit as $M$ goes to $+\infty$, we finally get that $U \in L^{q\left(p^{*}-1\right)}\left(\mathbb{R}^{N}\right)$ for some $q>N /(s p)$, together with the estimate

$$
\left\|U^{p^{*}-1}\right\|_{q}^{q} \leq\left(2 K_{0}^{\alpha-1}\left(\frac{p+\alpha-1}{p}\right)^{p}\right)^{\frac{p^{*}}{p}},
$$

and thus the conclusion.

Proposition 3.3 (Borderline Lorentz estimate). Let $U \in D^{s, p}\left(\mathbb{R}^{N}\right)$ be a non-negative solution of (3.1). Then

$$
U \in L^{q}\left(\mathbb{R}^{N}\right), \quad \text { for every } q>q_{0}:=\frac{(p-1) N}{N-s p} .
$$

\footnotetext{
${ }^{2}$ Here we use that

$$
G_{\alpha, M}(t) \geq \frac{p}{p+\alpha-1} t \min \{t, M\}^{\frac{\alpha-1}{p}} .
$$
}


Moreover, we have $U \in L^{q_{0}, \infty}\left(\mathbb{R}^{N}\right)$ with the estimate

$$
\sup _{t>0} t|\{U>t\}|^{\frac{1}{q_{0}}} \leq\|U\|_{p^{*}-1}^{\frac{p^{*}-1}{p-1}} .
$$

Proof. We divide the proof in two parts: we first prove (3.5). Then we will use (3.5) to prove (3.6).

Part I: intermediate estimate. Given $0<\alpha<1$ and $\varepsilon>0$, we take the Lipschitz increasing function $\psi_{\varepsilon}:[0,+\infty) \rightarrow[0,+\infty)$ defined as

$$
\psi_{\varepsilon}(t)=\int_{0}^{t}\left[(\varepsilon+\tau)^{\frac{\alpha-1}{p}}+\frac{\alpha-1}{p} \tau(\varepsilon+\tau)^{\frac{\alpha-1-p}{p}}\right]^{p} d \tau .
$$

We observe that

$$
0 \leq \psi_{\varepsilon}(t) \leq \int_{0}^{t}(\varepsilon+t)^{\alpha-1} d \tau=\frac{1}{\alpha}\left[(\varepsilon+t)^{\alpha}-\varepsilon^{\alpha}\right] \leq \frac{t^{\alpha}}{\alpha}
$$

where in the second inequality we used that $0<\alpha<1$. We insert in (3.2) the test function $\varphi=\psi_{\varepsilon}(U) \in D^{s, p}\left(\mathbb{R}^{N}\right)$. This gives

$$
\int_{\mathbb{R}^{2 N}} \frac{J_{p}(U(x)-U(y))\left(\psi_{\varepsilon}(U(x))-\psi_{\varepsilon}(U(y))\right.}{|x-y|^{N+s p}} d x d y=\mathcal{S}_{p, s} \int_{\mathbb{R}^{N}} U^{p^{*}-1} \psi_{\varepsilon}(U) d x .
$$

By defining

$$
\Psi_{\varepsilon}(t):=\int_{0}^{t} \psi_{\varepsilon}^{\prime}(\tau)^{\frac{1}{p}} d \tau=t(\varepsilon+t)^{\frac{\alpha-1}{p}},
$$

if we proceed as in the previous proof and use (3.7), we get

$$
\begin{aligned}
\left(\int_{\mathbb{R}^{N}} \Psi_{\varepsilon}(U)^{p^{*}} d x\right)^{\frac{p}{p^{*}}} \leq & \frac{1}{\alpha}\|U\|_{\infty}^{p^{*}+\alpha-1}\left|\left\{U>K_{0}\right\}\right| \\
& +\left(\int_{\left\{U \leq K_{0}\right\}} U^{p^{*}} d x\right)^{\frac{p^{*}-p}{p^{*}}}\left(\int_{\left\{U \leq K_{0}\right\}}\left(\psi_{\varepsilon}(U) U^{p-1}\right)^{\frac{p^{*}}{p}} d x\right)^{\frac{p}{p^{*}}},
\end{aligned}
$$

for $K_{0}>0$. Observe that we also used the previous Proposition to assure that $U \in L^{\infty}\left(\mathbb{R}^{N}\right)$. From (3.7) we get

$$
0 \leq \psi_{\varepsilon}(t) t^{p-1} \leq \frac{1}{\alpha}\left[(\varepsilon+t)^{\alpha}-\varepsilon^{\alpha}\right] t^{p-1} \leq \frac{1}{\alpha}(\varepsilon+t)^{\alpha-1} t^{p}=\frac{1}{\alpha} \Psi_{\varepsilon}(t)^{p} .
$$

Thus we arrive at

$$
\begin{aligned}
\left(\int_{\mathbb{R}^{N}} \Psi_{\varepsilon}(U)^{p^{*}} d x\right)^{\frac{p}{p^{*}}} & \leq \frac{1}{\alpha}\|U\|_{\infty}^{p^{*}+\alpha-1}\left|\left\{U>K_{0}\right\}\right| \\
& +\frac{1}{\alpha}\left(\int_{\left\{U \leq K_{0}\right\}} U^{p^{*}} d x\right)^{\frac{p^{*}-p}{p^{*}}}\left(\int_{\mathbb{R}^{N}} \Psi_{\varepsilon}(U)^{p^{*}} d x\right)^{\frac{p}{p^{*}}}
\end{aligned}
$$

The level $K_{0}=K_{0}(\alpha, U)>0$ is now chosen so that

$$
\left(\int_{\left\{U \leq K_{0}\right\}} U^{p^{*}} d x\right)^{\frac{p^{*}-p}{p^{*}}} \leq \frac{\alpha}{2},
$$


which yields

$$
\left(\int_{\mathbb{R}^{N}}\left(U(U+\varepsilon)^{\frac{\alpha-1}{p}}\right)^{p^{*}} d x\right)^{\frac{p}{p^{*}}} \leq \frac{2}{\alpha}\|U\|_{\infty}^{p^{*}+\alpha-1}\left|\left\{U>K_{0}\right\}\right|,
$$

for every $0<\alpha<1$. By taking the limit as $\varepsilon$ goes to 0 , we get the desired integrability (3.5). Part II: borderline Lorentz estimate. We now prove (3.6). For any $t>0$ we let $g_{t}(s)=$ $\min \{t, s\}$, and define

$$
G_{t}(s)=\int_{0}^{s} g_{t}^{\prime}(\tau)^{\frac{1}{p}} d \tau=g_{t}(s) .
$$

We test (3.2) with $g_{t}(U)$ and, thanks to [5, Lemma A.2] and Sobolev inequality we get

$$
\begin{aligned}
\mathcal{S}_{p, s}\left\|g_{t}(U)\right\|_{p^{*}}^{p} & \leq\left[g_{t}(U)\right]_{s, p}^{p} \leq \int_{\mathbb{R}^{2 N}} \frac{J_{p}(U(x)-U(y))\left(g_{t}(U(x))-g_{t}(U(y))\right)}{|x-y|^{N+s p}} d x d y \\
& \leq \mathcal{S}_{p, s} \int_{\mathbb{R}^{N}} U^{p^{*}-1} g_{t}(U) d x .
\end{aligned}
$$

We have $U \in L^{p^{*}-1}\left(\mathbb{R}^{N}\right)$, by choosing $q=p^{*}-1$ in (3.5). Thus we conclude that

$$
t|\{U>t\}|^{\frac{1}{p^{*}}} \leq\left\|g_{t}(U)\right\|_{p^{*}} \leq\left(\int_{\mathbb{R}^{N}} U^{p^{*}-1} g_{t}(U) d x\right)^{\frac{1}{p}} \leq t^{\frac{1}{p}}\|U\|_{p^{*}-1}^{\frac{p^{*}-1}{p}} .
$$

This finally yields (3.6), after some elementary manipulations.

3.2. Decay estimates. As an intermediate step towards the proof of the asymptotic result (1.11), in this subsection we will prove that any (positive) solution of (3.1) verifies

$$
\frac{1}{C}|x|^{-\frac{N-s p}{p-1}} \leq U(x) \leq C|x|^{-\frac{N-s p}{p-1}}, \quad|x|>1,
$$

for some $C=C(N, p, s, U)>1$, see Corollary 3.7 below.

In what follows, we will set for simplicity

$$
\Gamma(x)=|x|^{-\frac{N-s p}{p-1}}, \quad x \in \mathbb{R}^{N} \backslash\{0\},
$$

and

$$
\widetilde{\Gamma}(x)=\min \{1, \Gamma(x)\}=\min \left\{1,|x|^{-\frac{N-s p}{p-1}}\right\}, \quad x \in \mathbb{R}^{N} .
$$

The following expedient result will be useful.

Lemma 3.4. With the notation above, we have

$$
\frac{1}{C}|x|^{-N-s p} \leq\left(-\Delta_{p}\right)^{s} \widetilde{\Gamma}(x) \leq C|x|^{-N-s p}, \quad \text { for }|x|>R>1,
$$

in weak sense, for some $C=C(N, p, s, R)>1$. The constant blows-up as $R$ goes to 1 .

Proof. From Theorem A.4, we know that $\Gamma$ belongs to $\widetilde{D}^{s, p}\left(B_{R}^{c}\right)$ and is a weak solution of $\left(-\Delta_{p}\right)^{s} u=0$ in $B_{R}^{c}$ for any $R>1$. We then observe that the truncated function $\widetilde{\Gamma}$ can be written as

$$
\widetilde{\Gamma}(x)=\Gamma(x)-(\Gamma(x)-1)_{+} .
$$

Thus we apply Proposition 2.8, with the choices

$$
\Omega=B_{R}^{c}, \quad u=\Gamma, \quad f \equiv 0, \quad v=-(\Gamma-1)_{+},
$$


This yields for $|x|>R$

$$
\begin{aligned}
\left(-\Delta_{p}\right)^{s} \widetilde{\Gamma}(x) & =2 \int_{B_{1}} \frac{J_{p}(\Gamma(x)-1)-J_{p}(\Gamma(x)-\Gamma(y))}{|x-y|^{N+s p}} d y \\
& =2 \int_{B_{1}} \frac{J_{p}(\Gamma(y)-\Gamma(x))-J_{p}(1-\Gamma(x))}{|x-y|^{N+s p}} d y .
\end{aligned}
$$

We first prove the upper bound in (3.9). To this aim, by the monotonicity of $\Gamma$ we get

$$
(\Gamma(y)-\Gamma(x))^{p-1}-(1-\Gamma(x))^{p-1} \leq(\Gamma(y)-\Gamma(x))^{p-1} \leq \Gamma(y)^{p-1}, \quad|x|>R,|y| \leq 1 .
$$

Moreover

$$
|x-y| \geq \frac{R-1}{R}|x|, \quad \text { for all }|x|>R \text { and }|y|<1 .
$$

By spending these informations in (3.10), we obtain

$$
\left(-\Delta_{p}\right)^{s} \widetilde{\Gamma}(x) \leq\left(\frac{R}{R-1}\right)^{N+s p} \frac{2}{|x|^{N+s p}} \int_{B_{1}} \Gamma(y)^{p-1} d y=\frac{C}{|x|^{N+s p}},
$$

as desired. Observe that we also used that $\Gamma \in L_{\text {loc }}^{p-1}\left(\mathbb{R}^{N}\right)$.

In order to prove the lower bound, we need to distinguish between the case $1<p<2$ and the case $p \geq 2$. If $p \geq 2$, then $J_{p}(t)=|t|^{p-2} t$ is a convex superadditive function on $[0, \infty)$. Thus we get

$$
J_{p}(\Gamma(y)-\Gamma(x))-J_{p}(1-\Gamma(x)) \geq J_{p}(\Gamma(y)-1), \quad|x|>R>1>|y| .
$$

As for the kernel, we have

$$
|x-y|<2|x|, \quad \text { if }|x|>|y|,
$$

thus in conclusion from $(3.10)$ we get

$$
\left(-\Delta_{p}\right)^{s} \widetilde{\Gamma}(x) \geq \frac{2^{1-N-s p}}{|x|^{N+s p}} \int_{B_{1}}(\Gamma(y)-1)^{p-1} d y=\frac{C}{|x|^{N+s p}} .
$$

Using again that $\Gamma \in L_{\text {loc }}^{p-1}\left(\mathbb{R}^{N}\right)$ and that $\Gamma>1$ in $B_{1}$ gives the lower bound in (3.9), in the case $p \geq 2$.

In the case $1<p<2$, we need to use (2.3), which gives

$$
\begin{aligned}
J_{p}(\Gamma(y)-\Gamma(x))-J_{p}(1-\Gamma(x)) & \geq C \frac{(\Gamma(y)-1)}{\left((\Gamma(y)-\Gamma(x))^{2}+(1-\Gamma(x))^{2}\right)^{\frac{2-p}{2}}} \\
& \geq \frac{C}{2^{\frac{2-p}{2}}} \frac{(\Gamma(y)-1)}{(\Gamma(y)-\Gamma(x))^{2-p}} \geq C \Gamma(y)^{p-1}\left(1-\frac{1}{\Gamma(y)}\right) .
\end{aligned}
$$

By using this and (3.11) in (3.10), we get the desired lower bound for $1<p<2$ as well.

In order to prove a lower bound for positive radially decreasing solutions of (3.1), we need to focus on the auxiliary problem

$$
\mathcal{I}(R)=\inf _{u \in D^{s, p}\left(\mathbb{R}^{N}\right)}\left\{[u]_{s, p}^{p}: u \geq \chi_{B_{R}}\right\} .
$$


Proposition 3.5. Let $1<p<\infty$ and $s \in(0,1)$ be such that $s p<N$. For any $R>0$, problem (3.12) has a unique solution $u_{R}>0$. Moreover, $u_{R}$ is radial, non-increasing and $u_{R} \in D^{s, p}\left(\mathbb{R}^{N}\right)$ solves in weak sense

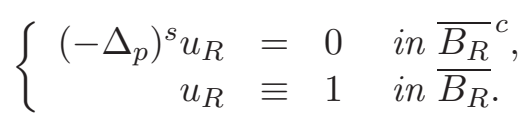

Proof. The existence of a solution follows easily by using the Direct Methods. Indeed, if $\left\{u_{n}\right\}_{n \in \mathbb{N}} \subset D^{s, p}\left(\mathbb{R}^{N}\right)$ is a minimizing sequence, then a uniform bound on their Gagliardo seminorms entails a uniform bound on the $L^{p^{*}}$ norms, by Sobolev inequality. Thus we have weak convergence (up to a subsequence) in $L^{p^{*}}\left(\mathbb{R}^{N}\right)$ to a function $u \in D^{s, p}\left(\mathbb{R}^{N}\right)$. Moreover, the constraint $u_{n} \geq \chi_{B_{R}}$ is stable with respect to weak convergence and thus it passes to the limit. Consequently, $u$ is a minimizer. The uniqueness follows from strict convexity of the functional.

All the other required properties of $u_{R}$ follow as in the proof of Proposition 3.1, we just show that $u_{R}$ saturates the constraint $u_{R} \geq \chi_{B_{R}}$. For simplicity, we set $\mathcal{E}(u)=[u]_{s, p}^{p}$. Then from [16, Remark 3.3] we have

$$
\mathcal{E}(\max \{u, t\})+\mathcal{E}(\min \{u, t\}) \leq \mathcal{E}(u), \quad \text { for every } u \in D^{s, p}\left(\mathbb{R}^{N}\right), t \in \mathbb{R} .
$$

In particular, $\min \left\{u_{R}, 1\right\}$ is admissible and is still a minimizer. Thus by uniqueness it coincides with $u_{R}$.

Thanks to Lemma 3.4, we can prove a decay estimate for the solution of (3.12).

Proposition 3.6. The solution $u_{1}$ of problem (3.12) with $R=1$ satisfies

$$
\frac{|x|^{-\frac{N-s p}{p-1}}}{C} \leq u_{1}(x) \leq p^{\frac{1}{p-1}}|x|^{-\frac{N-s p}{p-1}}, \quad \text { for }|x| \geq 1,
$$

for some constant $C=C(N, p, s)>1$.

Proof. Observe that $u_{1}$ is continuous due to [19, Theorem 1.1]. We prove the two estimates separately.

Upper bound. We first observe that by using the scaling properties of the Gagliardo seminorm, we have

$$
\mathcal{I}(R)=R^{N-s p} \mathcal{I}(1) .
$$

For every $R>1$, we set $u_{1}(R)=t \in(0,1)$. As in the previous proof, we set $\mathcal{E}(u)=[u]_{s, p}^{p}$. The function $\min \left\{u_{1}, t\right\} / t$ is admissible for problem (3.12) with $B_{R}$, then the minimality of $u_{R}$ gives

$$
\mathcal{E}\left(\frac{\min \left\{u_{1}, t\right\}}{t}\right) \geq \mathcal{E}\left(u_{R}\right)=\mathcal{I}(R)=R^{N-s p} \mathcal{I}(1)
$$

thanks to (3.14). Similarly, we get

$$
\mathcal{E}\left(\frac{\max \left\{u_{1}-t, 0\right\}}{1-t}\right) \geq \mathcal{E}\left(u_{1}\right)=\mathcal{I}(1)
$$

then using the $p$-homogeneity of the energy and summing the previous two inequalities

$$
\mathcal{E}\left(\min \left\{u_{1}, t\right\}\right)+\mathcal{E}\left(\max \left\{u_{1}, t\right\}\right) \geq\left(t^{p} R^{N-s p}+(1-t)^{p}\right) \mathcal{I}(1) .
$$


Using the submodularity of Gagliardo seminorms (3.13) in the left-hand side and simplifying we get

$$
t^{p} R^{N-s p} \leq 1-(1-t)^{p} .
$$

By recalling the definition of $t$, we obtain

$$
u_{1}(R)^{p} R^{N-s p} \leq 1-\left(1-u_{1}(R)\right)^{p}
$$

and since $1-\left(1-u_{1}(R)\right)^{p} \leq p u_{1}(R)$ we get

$$
u_{1}(R) \leq p^{\frac{1}{p-1}} R^{-\frac{N-s p}{p-1}} .
$$

Lower bound. By using Proposition 2.8 with

$$
\Omega=\bar{B}_{3}^{c}, \quad u=u_{1}, \quad f \equiv 0, \quad v=-\left(u_{1}-u_{1}(2)\right)_{+},
$$

the truncated function

$$
u=\min \left\{u_{1}, u_{1}(2)\right\}=u_{1}-\left(u_{1}-u_{1}(2)\right)_{+},
$$

satisfies weakly in ${\overline{B_{3}}}^{c}$

$$
\begin{aligned}
\left(-\Delta_{p}\right)^{s} u(x) & =2 \int_{B_{2}} \frac{J_{p}\left(u_{1}(x)-u_{1}(2)\right)-J_{p}\left(u_{1}(x)-u_{1}(y)\right)}{|x-y|^{N+s p}} d y \\
& \geq 2 \int_{B_{1}} \frac{J_{p}\left(u_{1}(y)-u_{1}(x)\right)-J_{p}\left(u_{1}(2)-u_{1}(x)\right)}{|x-y|^{N+s p}} d y .
\end{aligned}
$$

In the last passage we used that the integrand is nonnegative by the monotonicity of $u_{1}$. Recall that $u_{1} \equiv 1$ in $B_{1}$ and by (3.15) we have $u_{1}(2)<1=u_{1}(1)$. Then, it is readily checked that

$$
\left(u_{1}(1)-u_{1}(x)\right)^{p-1}-\left(u_{1}(2)-u_{1}(x)\right)^{p-1} \geq c, \quad \text { for }|x|>3,
$$

for some constant $c=c\left(p, u_{1}(1)-u_{1}(2)\right)>0$. Since also $|x-y| \leq 2|x|$ for all $x \in B_{2}^{c}$ and $y \in B_{1}$, the previous discussion yields

$$
\left(-\Delta_{p}\right)^{s} u(x) \geq \frac{2 c\left|B_{1}\right|}{(2|x|)^{N+s p}}=: \frac{c_{1}}{|x|^{N+s p}}, \quad \text { for }|x|>3 .
$$

On the other hand, from Lemma 3.4, for every $\varepsilon>0$ we have

$$
\left(-\Delta_{p}\right)^{s}(\varepsilon \widetilde{\Gamma}(x)) \leq \frac{c_{2}}{|x|^{N+s p}} \varepsilon^{p-1}, \quad \text { for }|x|>3
$$

where $\widetilde{\Gamma}$ is given in (3.8). Now choose $\varepsilon>0$ as follows

$$
\varepsilon=\min \left\{u_{1}(3) 3^{\frac{N-s p}{p-1}},\left(\frac{c_{1}}{c_{2}}\right)^{\frac{1}{p-1}}\right\},
$$

so that by (3.16) and (3.17) it holds

$$
\left\{\begin{array}{rlrl}
\left(-\Delta_{p}\right)^{s}(\varepsilon \widetilde{\Gamma}) & \leq\left(-\Delta_{p}\right)^{s} u & & \text { in }{\overline{B_{3}}}^{c}, \\
\varepsilon \widetilde{\Gamma} \leq u & & \text { in } \overline{B_{3} .}
\end{array}\right.
$$

Therefore by Theorem 2.7 and the definitions of $\widetilde{\Gamma}$ and $u$ we have

$$
\varepsilon|x|^{-\frac{N-s p}{p-1}} \leq u(x)=u_{1}(x), \quad \text { for }|x|>3 .
$$


In $\overline{B_{3}} \backslash B_{1}$ the estimate is simpler to obtain, indeed

$$
u_{1}(3)|x|^{-\frac{N-s p}{p-1}} \leq u_{1}(3) \leq u_{1}(x)
$$

thus we get the conclusion.

Finally, we can prove the aforementioned decay estimate for solutions of (3.1).

Corollary 3.7 (Sharp decay rate). Let $U \in D^{s, p}\left(\mathbb{R}^{N}\right)$ be a positive radially symmetric and decreasing solution of (3.1). Then

$$
\left(\inf _{B_{1}} U\right) \frac{|x|^{-\frac{N-s p}{p-1}}}{C} \leq U(x) \leq\left(\omega_{N}^{-\frac{1}{p^{*}}}\|U\|_{p^{*}-1}^{\frac{p^{*}-1}{p}}\right)^{\frac{p}{p-1}}|x|^{-\frac{N-s p}{p-1}}, \quad|x| \geq 1,
$$

where the constant $C=C(N, p, s)>1$ is the same of Proposition 3.6.

Proof. The upper bound follows from the borderline $L^{q_{0}, \infty}$ estimate of (3.6), combined with the Radial Lemma 2.9.

As for the lower bound, by the weak Harnack inequality for positive supersolution of $\left(-\Delta_{p}\right)^{s}$ (see [19, Theorem 5.2]), we have

$$
\lambda:=\inf _{B_{1}} U \geq C\left(\int_{B_{2}} U^{p-1} d x\right)^{\frac{1}{p-1}}>0 .
$$

Then the function $\lambda u_{1}$ is a lower barrier for $U$ in $B_{1}^{c}$. Thus the lower bound follows from Theorem 2.7 and Proposition 3.6.

\section{Proof of the main Result}

In this section we still denote by $\widetilde{\Gamma}$ the truncated function defined by (3.8), while $U$ is a positive radially symmetric and decreasing solution of (3.1). As in the previous section we will systematically use the abuse of notation $U(x)=U(r)$ and $\widetilde{\Gamma}(x)=\widetilde{\Gamma}(r)$, for $r=|x|$.

Lemma 4.1. Suppose that

$$
U(R) \geq A \widetilde{\Gamma}(R) \quad \text { for some } R>2 .
$$

For any $\delta>0$ there exists $\theta=\theta(N, p, s, \delta, U)<1$ such that

$$
U(r) \geq(A-\delta) \widetilde{\Gamma}(r) \quad \text { for any } \theta R \leq r \leq R .
$$

Similarly, if

$$
U(R) \leq B \widetilde{\Gamma}(R) \quad \text { for some } R>2,
$$

then

$$
U(r) \leq(B+\delta) \widetilde{\Gamma}(r) \quad \text { for any } R \leq r \leq R / \theta .
$$

Proof. Consider the first statement and let $\theta<1$ to be determined. $U$ is non increasing and

$$
U(r) \leq C r^{-\frac{N-s p}{p-1}}, \quad r \geq 1,
$$

by Corollary 3.7. Then for any $\theta R \leq r \leq R$ it holds

$$
\frac{U(R)}{\widetilde{\Gamma}(R)}-\frac{U(r)}{\widetilde{\Gamma}(r)} \leq U(R)\left(\frac{1}{\widetilde{\Gamma}(R)}-\frac{1}{\widetilde{\Gamma}(r)}\right) \leq \frac{C}{R^{\frac{N-s p}{p-1}}}\left(R^{\frac{N-s p}{p-1}}-r^{\frac{N-s p}{p-1}}\right) \leq C\left(1-\theta^{\frac{N-s p}{p-1}}\right) .
$$


Therefore by hypothesis we get

$$
\frac{U(r)}{\widetilde{\Gamma}(r)} \geq A-C\left(1-\theta^{\frac{N-s p}{p-1}}\right), \quad \text { for } r \in[\theta R, R],
$$

which gives the first claim. The proof of the other statement is similar: for any $R \leq r \leq R / \theta$ it holds

$$
\frac{U(r)}{\widetilde{\Gamma}(r)}-\frac{U(R)}{\widetilde{\Gamma}(R)} \leq U(R)\left(\frac{1}{\widetilde{\Gamma}(r)}-\frac{1}{\widetilde{\Gamma}(R)}\right) \leq \frac{C}{R^{\frac{N-s p}{p-1}}}\left(r^{\frac{N-s p}{p-1}}-R^{\frac{N-s p}{p-1}}\right) \leq C\left(\theta^{-\frac{N-s p}{p-1}}-1\right)
$$

which gives

$$
\frac{U(r)}{\widetilde{\Gamma}(r)} \leq B+C\left(\theta^{-\frac{N-s p}{p-1}}-1\right), \quad \text { for } r \in[R, R / \theta] .
$$

This completes the proof.

We are ready for the proof of the main result.

Theorem 4.2. There exists $U_{\infty}>0$ such that

$$
\lim _{r \rightarrow+\infty} r^{\frac{N-s p}{p-1}} U(r)=U_{\infty} .
$$

Proof. We can suppose that $p \neq 2$, since for $p=2$ the function $U$ has an explicit expression. By virtue of Corollary 3.7 we readily have

$$
\frac{1}{C} \leq m:=\liminf _{r \rightarrow+\infty} \frac{U(r)}{\widetilde{\Gamma}(r)} \leq \limsup _{r \rightarrow+\infty} \frac{U(r)}{\widetilde{\Gamma}(r)}=: M \leq C,
$$

with $C$ depending on $U$ as well. Suppose by contradiction that $M-m>0$, and fix $0<\varepsilon_{0}<$ $(M-m) / 4$.

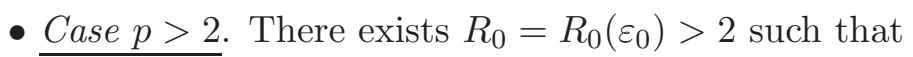

$$
\frac{U(r)}{\widetilde{\Gamma}(r)} \geq m-\varepsilon_{0}, \quad \text { for } r \geq R_{0},
$$

and we can choose an arbitrarily large $R>R_{0}$ such that

$$
\frac{U(R)}{\widetilde{\Gamma}(R)} \geq M-\frac{M-m}{4}
$$

Consider $\delta=(M-m) / 4$. By Lemma 4.1, there exists $\theta<1$ so that for any such $R$ it holds

$$
\frac{U(r)}{\widetilde{\Gamma}(r)} \geq \frac{M+m}{2}, \quad \text { for } r \in[\theta R, R]
$$

Since $R$ can be chosen arbitrarily large, we can suppose $\theta R>R_{0}$ as well. Consider, for any $0<\varepsilon<(M-m) / 4$, the lower barrier $w(r)=g(r) \widetilde{\Gamma}(r)$ where $g$ is the following step function

$$
g(r)= \begin{cases}0 & \text { if } r<R_{0}, \\ m-\varepsilon_{0} & \text { if } R_{0} \leq r<\theta R \\ \frac{M+m}{2} & \text { if } \theta R \leq r<\sqrt{\theta} R \\ m+\varepsilon & \text { if } \sqrt{\theta} R<r\end{cases}
$$


It is easily seen that ${ }^{3} w \in \widetilde{D}^{s, p}\left({\overline{B_{R}}}^{c}\right)$. Moreover, by using (4.1), it is readily verified that $w \leq U$ in $\overline{B_{R}}$. We claim that, for sufficiently small $\varepsilon_{0}$ and $\varepsilon$ and sufficiently large $R$, it holds

$$
\left(-\Delta_{p}\right)^{s} w \leq\left(-\Delta_{p}\right)^{s} U, \quad \text { in }{\overline{B_{R}}}^{c} \text {. }
$$

This would end the proof, since Theorem 2.7 would yield $U \geq w$ in $\mathbb{R}^{N}$ and then

$$
m=\liminf _{r \rightarrow+\infty} r^{\frac{N-s p}{p-1}} U(r)=\liminf _{r \rightarrow+\infty} \frac{U(r)}{\widetilde{\Gamma}(r)} \geq \liminf _{r \rightarrow+\infty} g(r)=m+\varepsilon,
$$

giving a contradiction. The function $w-(m+\varepsilon) \widetilde{\Gamma}$ is supported in $B_{\sqrt{\theta} R} \Subset B_{R}$ and thus using Proposition 2.8 with

$$
\Omega=\bar{B}_{R}^{c}, \quad u=(m+\varepsilon) \widetilde{\Gamma}, \quad f=\left(-\Delta_{p}\right)^{s}((m+\varepsilon) \widetilde{\Gamma}), \quad v=w-(m+\varepsilon) \widetilde{\Gamma},
$$

and (3.9), for any $|x|>R$ it holds

$$
\begin{aligned}
\left(-\Delta_{p}\right)^{s} w(x)= & (m+\varepsilon)^{p-1}\left(-\Delta_{p}\right)^{s} \widetilde{\Gamma}(x) \\
& +\int_{B_{\sqrt{\theta} R}} \frac{J_{p}((m+\varepsilon) \widetilde{\Gamma}(x)-w(y))-J_{p}((m+\varepsilon)(\widetilde{\Gamma}(x)-\widetilde{\Gamma}(y)))}{|x-y|^{N+s p}} d y \\
\leq & \frac{C}{|x|^{N+s p}}+\int_{B_{\sqrt{\theta} R}} \frac{h(x, y)}{|x-y|^{N+s p}} d y
\end{aligned}
$$

where

$$
h(x, y)=J_{p}((m+\varepsilon)(\widetilde{\Gamma}(y)-\widetilde{\Gamma}(x)))-J_{p}(w(y)-(m+\varepsilon) \widetilde{\Gamma}(x)) .
$$

We now decompose the last integral in (4.2) as follows

$$
\int_{B_{\sqrt{\theta} R}} d y=\int_{B_{R_{0}}} d y+\int_{B_{\theta R} \backslash B_{R_{0}}} d y+\int_{B_{\sqrt{\theta} R} \backslash B_{\theta R}} d y
$$

and proceed to estimate each term separately.

Being $R_{0}=R_{0}\left(\varepsilon_{0}\right)$ and $h$ universally bounded, it holds

$$
\int_{B_{R_{0}}} \frac{h(x, y)}{|x-y|^{N+s p}} d y \leq\|h\|_{L^{\infty}\left(\mathbb{R}^{2 N}\right)} \frac{\omega_{N} R_{0}^{N}}{|| x\left|-R_{0}\right|^{N+s p}} \leq \frac{C\left(\varepsilon_{0}\right)}{|x|^{N+s p}}(1-\theta)^{-N-s p},
$$

where we used that (recall that we are assuming $\theta R>R_{0}$ )

$$
|| x\left|-R_{0}\right| \geq\left(1-\frac{R_{0}}{R}\right)|x| \geq(1-\theta)|x|, \quad \text { for }|x|>R .
$$

For the second integral in (4.3), we notice that for $y \in B_{\theta R} \backslash B_{R_{0}}$ and $x \in B_{R}^{c}$ we have

$$
h(x, y)=J_{p}((m+\varepsilon)(\widetilde{\Gamma}(y)-\widetilde{\Gamma}(x)))-J_{p}\left((m+\varepsilon)(\widetilde{\Gamma}(y)-\widetilde{\Gamma}(x))-\left(\varepsilon+\varepsilon_{0}\right) \widetilde{\Gamma}(y)\right) .
$$

Observe that by (2.1), with simple manipulations we get

$$
h(x, y) \leq c\left[(m+\varepsilon)^{p-2}+\left(\varepsilon+\varepsilon_{0}\right)^{p-2}\right]\left(\varepsilon+\varepsilon_{0}\right) \widetilde{\Gamma}(y),
$$

for $x \in B_{R}^{c}, y \in B_{\theta R} \backslash B_{R_{0}}$ and $c=c(p)>0$. Therefore, since

$$
|x-y| \geq|| x|-| y|| \geq|x|-\theta R \geq(1-\theta)|x|, \quad \text { for } x \in B_{R}^{c}, y \in B_{\theta R},
$$

\footnotetext{
${ }^{3}$ As a set $E$ occuring in the definition $\widetilde{D}^{s, p}\left({\overline{B_{R}}}^{c}\right)$ one can take for example $E=\bar{B}_{\sqrt{\theta}}^{c}$.
} 
recalling the definition of $\widetilde{\Gamma}$ we get

$$
\begin{aligned}
\int_{B_{\theta R} \backslash B_{R_{0}}} \frac{h(x, y)}{|x-y|^{N+s p}} d y & \leq \frac{C\left(\varepsilon+\varepsilon_{0}\right)}{(1-\theta)^{N+s p}|x|^{N+s p}} \int_{B_{\theta R} \backslash B_{R_{0}}} \frac{1}{|y|^{N-s p}} d y \\
& \leq C \frac{\left(\varepsilon+\varepsilon_{0}\right)}{(1-\theta)^{N+s p}} \frac{(\theta R)^{s p}}{|x|^{N+s p}},
\end{aligned}
$$

where $C=C(N, s, p, M+m)>0$. For the third integral in (4.3), for $y \in B_{\sqrt{\theta} R} \backslash B_{\theta R}$ we have

$$
\begin{aligned}
h(x, y) & =J_{p}((m+\varepsilon)(\widetilde{\Gamma}(y)-\widetilde{\Gamma}(x)))-J_{p}\left((m+\varepsilon)(\widetilde{\Gamma}(y)-\widetilde{\Gamma}(x))+\left(\frac{M-m}{2}-\varepsilon\right) \widetilde{\Gamma}(y)\right) \\
& \leq J_{p}(\underbrace{(m+\varepsilon)(\widetilde{\Gamma}(y)-\widetilde{\Gamma}(x))}_{a})-J_{p}(\underbrace{(m+\varepsilon)(\widetilde{\Gamma}(y)-\widetilde{\Gamma}(x))}_{a}+\underbrace{\left(\frac{M-m}{4}\right) \widetilde{\Gamma}(y)}_{b}),
\end{aligned}
$$

since $\varepsilon<(M-m) / 4$. The inequality (2.2) thus gives

$$
h(x, y) \leq-2^{2-p}\left(\frac{M-m}{4}\right)^{p-1} \widetilde{\Gamma}(y)^{p-1} .
$$

Therefore, using

$$
|x-y| \leq 2|x|, \quad \text { for }|x|>R,|y|<\sqrt{\theta} R,
$$

we obtain

$$
\begin{aligned}
\int_{B_{\sqrt{\theta} R} \backslash B_{\theta R}} \frac{h(x, y)}{|x-y|^{N+s p}} d y & \leq-\frac{c(M-m)^{p-1}}{|x|^{N+s p}} \int_{B_{\sqrt{\theta} R} \backslash B_{\theta R}}|y|^{s p-N} d y \\
& \leq-c \theta^{\frac{s p}{2}}\left(1-\theta^{\frac{s p}{2}}\right)(M-m)^{p-1} \frac{R^{s p}}{|x|^{N+s p}}
\end{aligned}
$$

for a constant $c=c(N, s, p)>0$. Gathering toghether the estimates (4.2), (4.4), (4.5) and (4.6) we proved

$$
\begin{aligned}
\left(-\Delta_{p}\right)^{s} w(x) \leq & \left(C+\frac{C\left(\varepsilon_{0}\right)}{(1-\theta)^{N+s p}}\right) \frac{1}{|x|^{N+s p}} \\
& -\left[c\left(1-\theta^{\frac{s p}{2}}\right)(M-m)^{p-1}-\frac{C\left(\varepsilon+\varepsilon_{0}\right)}{(1-\theta)^{N+s p}}\right] \frac{R^{s p} \theta^{s p}}{|x|^{N+s p}} .
\end{aligned}
$$

Thus we can choose $\varepsilon+\varepsilon_{0}$ small enough (depending only on $N, p, s, M-m$ and the chosen minimizer $U$ ), so that the second term above is negative. For any such a choice we have, for any $|x|>R$,

$$
\left(-\Delta_{p}\right)^{s} w(x) \leq \frac{C\left(\varepsilon_{0}\right)}{|x|^{N+s p}}, \quad\left(-\Delta_{p}\right)^{s} U(x)=U(x)^{p^{*}-1} \geq \frac{1}{C|x|^{N+\frac{s p}{p-1}}},
$$

where in the last estimate we used Corollary 3.7. Since $p>2$, for sufficiently large $R$ it holds

$$
\frac{1}{C|x|^{N+\frac{s p}{p-1}}} \geq \frac{R^{s p \frac{p-2}{p-1}}}{C|x|^{N+s p}} \geq \frac{C\left(\varepsilon_{0}\right)}{|x|^{N+s p}},
$$

and thus the claim follows. 
- $\underline{\text { Case } 1<p<2}$. There exists $R_{0}=R_{0}\left(\varepsilon_{0}\right)>2$ such that

$$
\frac{U(r)}{\widetilde{\Gamma}(r)} \leq M+\varepsilon_{0}, \quad \text { for } r \geq R_{0}
$$

and we can choose an arbitrarily large $R>R_{0}$ such that

$$
\frac{U(R)}{\widetilde{\Gamma}(R)} \leq m+\frac{M-m}{4}
$$

As before, we consider $\delta=(M-m) / 4$ in Lemma 4.1: there exists $\theta<1$ so that for any such $R$ it holds

$$
\frac{U(r)}{\widetilde{\Gamma}(r)} \leq \frac{M+m}{2}, \quad \text { for every } r \in[R, R / \theta]
$$

Since $U \in L^{\infty}\left(\mathbb{R}^{N}\right)$, there exists $\bar{C}>0$ such that $U \leq \bar{C} \widetilde{\Gamma}$ in $\mathbb{R}^{N}$, then for any $0<\varepsilon<$ $(M-m) / 4$ we consider the upper barrier $w(r)=g(r) \widetilde{\Gamma}(r)$, where

$$
g(r)= \begin{cases}\bar{C} & \text { if } r<R_{0}, \\ M+\varepsilon_{0} & \text { if } R_{0} \leq r<R \\ \frac{M+m}{2} & \text { if } R \leq r<R / \sqrt{\theta} \\ M-\varepsilon & \text { if } R / \sqrt{\theta}<r .\end{cases}
$$

Again, it is easy to verify that $w \in \widetilde{D}^{s, p}\left({\overline{B_{R}}}^{c}\right)$. Using (4.7), we can verify that $w \geq U$ in $B_{R / \theta}$. We claim that, for sufficiently small $\varepsilon_{0}$ and $\varepsilon$ and sufficiently large $R$, it holds

$$
\left(-\Delta_{p}\right)^{s} w \geq\left(-\Delta_{p}\right)^{s} U, \quad \text { in } B_{R / \theta}^{c} .
$$

This would end the proof, since the comparison principle of Theorem 2.7 would yield $U \leq w$ in $\mathbb{R}^{N}$ and then

$$
M=\limsup _{r \rightarrow+\infty} r^{\frac{N-s p}{p-1}} U(r)=\limsup _{r \rightarrow+\infty} \frac{U(r)}{\widetilde{\Gamma}(r)} \leq \limsup _{r \rightarrow+\infty} g(r)=M-\varepsilon,
$$

which gives again a contradiction. The function $w-(M-\varepsilon) \widetilde{\Gamma}$ is supported in $B_{R / \sqrt{\theta}} \Subset B_{R / \theta}$ and thus using again Proposition 2.8 with

$$
\Omega={\overline{B_{R / \theta}}}^{c}, \quad u=(M-\varepsilon) \widetilde{\Gamma}, \quad f=\left(-\Delta_{p}\right)^{s}((M-\varepsilon) \widetilde{\Gamma}), \quad v=w-(M-\varepsilon) \widetilde{\Gamma},
$$

and (3.9), for any $|x|>R / \theta$ it holds

$$
\begin{aligned}
\left(-\Delta_{p}\right)^{s} w(x)= & (M-\varepsilon)^{p-1}\left(-\Delta_{p}\right)^{s} \widetilde{\Gamma}(x) \\
& +\int_{B_{R / \sqrt{\theta}}} \frac{J_{p}((M-\varepsilon) \widetilde{\Gamma}(x)-w(y))-J_{p}((M-\varepsilon)(\widetilde{\Gamma}(x)-\widetilde{\Gamma}(y)))}{|x-y|^{N+s p}} d y \\
\geq & \frac{1}{C|x|^{N+s p}}+\int_{B_{R / \sqrt{\theta}}} \frac{h(x, y)}{|x-y|^{N+s p}} d y,
\end{aligned}
$$

where

$$
h(x, y)=J_{p}((M-\varepsilon)(\widetilde{\Gamma}(y)-\widetilde{\Gamma}(x)))-J_{p}(w(y)-(M-\varepsilon) \widetilde{\Gamma}(x)) .
$$


As above, we now decompose the last integral in (4.8) as

$$
\int_{B_{R / \sqrt{\theta}}} d y=\int_{B_{R_{0}}} d y+\int_{B_{R} \backslash B_{R_{0}}} d y+\int_{B_{R / \sqrt{\theta} \backslash B_{R}}} d y,
$$

and proceed to estimate each term separately.

Being $R_{0}=R_{0}\left(\varepsilon_{0}\right)$ and $h$ universally bounded, as before we get

$$
\int_{B_{R_{0}}} \frac{h(x, y)}{|x-y|^{N+s p}} d y \geq-\frac{C\left(\varepsilon_{0}\right)}{|x|^{N+s p}}
$$

where this time we used that (recall that we are assuming $R>R_{0}$ )

$$
|| x\left|-R_{0}\right| \geq\left(1-\frac{R_{0}}{R} \theta\right)|x| \geq(1-\theta)|x|, \quad \text { for }|x|>R / \theta .
$$

For $y \in B_{R} \backslash B_{R_{0}}$ we have

$$
h(x, y)=J_{p}((M-\varepsilon)(\widetilde{\Gamma}(y)-\widetilde{\Gamma}(x)))-J_{p}\left((M-\varepsilon)(\widetilde{\Gamma}(y)-\widetilde{\Gamma}(x))+\left(\varepsilon+\varepsilon_{0}\right) \widetilde{\Gamma}(y)\right),
$$

and by subaddivity of $\tau \mapsto \tau^{p-1}$, we get

$$
h(x, y) \geq-\left(\varepsilon+\varepsilon_{0}\right)^{p-1} \widetilde{\Gamma}(y)^{p-1} .
$$

Therefore, the analogue of (4.5) is now

$$
\int_{B_{R} \backslash B_{R_{0}}} \frac{h(x, y)}{|x-y|^{N+s p}} d y \geq-C\left(\varepsilon+\varepsilon_{0}\right)^{p-1} \frac{R^{s p}}{|x|^{N+s p}},
$$

and again $C=C(N, s, p, M+m)>0$. For the previous estimate we also used that

$$
|x-y| \geq|x|-R \geq(1-\theta)|x|, \quad \text { for }|x|>R / \theta,|y|<R .
$$

For $y \in B_{R / \sqrt{\theta}} \backslash B_{R}$ and $x \in B_{R / \theta}^{c}$ we have

$$
\begin{aligned}
h(x, y) & =J_{p}(\underbrace{(M-\varepsilon)(\widetilde{\Gamma}(y)-\widetilde{\Gamma}(x))}_{a}) \\
& -J_{p}(\underbrace{(M-\varepsilon)(\widetilde{\Gamma}(y)-\widetilde{\Gamma}(x))}_{a}-\underbrace{\left(\frac{M-m}{2}-\varepsilon\right) \widetilde{\Gamma}(y)}_{b}) .
\end{aligned}
$$

Clearly

$$
0 \leq a=(M-\varepsilon)(\widetilde{\Gamma}(y)-\widetilde{\Gamma}(x)) \leq(M-\varepsilon) \widetilde{\Gamma}(y)=: A,
$$

so that (2.4) provides

$$
h(x, y) \geq \max \left\{(M-\varepsilon)^{p-1}-\left(\frac{M+m}{2}\right)^{p-1},\left(\frac{M-m}{2}-\varepsilon\right)^{p-1} 2^{1-p}\right\} \widetilde{\Gamma}(y)^{p-1} .
$$

Proceeding as for (4.6) and using

$$
|x-y| \leq 2|x|, \quad \text { for } x \in B_{R / \theta}^{c}, y \in B_{R / \sqrt{\theta}},
$$

we thus obtain

$$
\int_{B_{R / \sqrt{\theta}} \backslash B_{R}} \frac{h(x, y)}{|x-y|^{N+s p}} d y \geq \frac{c}{|x|^{N+s p}} \int_{B_{R / \sqrt{\theta}} \backslash B_{R}}|y|^{s p-N} d y \geq c \frac{R^{s p}}{|x|^{N+s p}},
$$


for a small constant $c$ depending only on $M$ and $m$. Gathering together the estimates (4.8), (4.9), (4.10) and (4.11), we proved

$$
\left(-\Delta_{p}\right)^{s} w(x) \geq-\frac{C\left(\varepsilon_{0}\right)}{|x|^{N+s p}}+\left(c-C\left(\varepsilon+\varepsilon_{0}\right)^{p-1}\right) \frac{R^{s p}}{|x|^{N+s p}} .
$$

in $B_{R / \theta}^{c}$. We can thus choose $\varepsilon_{0}$ and $\varepsilon$ small enough so that the second term above is positive. For any such choice we have, for any $|x|>R / \theta$,

$$
\left(-\Delta_{p}\right)^{s} w(x) \geq-\frac{C\left(\varepsilon_{0}\right)}{|x|^{N+s p}}+\frac{c}{2} \frac{R^{s p}}{|x|^{N+s p}},
$$

and for sufficiently large $R$ so that $c R^{s p}>4 C\left(\varepsilon_{0}\right)$ it holds

$$
\left(-\Delta_{p}\right)^{s} w(x) \geq \frac{c}{4} \frac{R^{s p}}{|x|^{N+s p}} .
$$

By using Corollary 3.7 and the fact that $1<p<2$, for every $|x| \geq R / \theta$ we get

$$
\left(-\Delta_{p}\right)^{s} U(x)=U^{p^{*}-1}(x) \leq \frac{C}{|x|^{N+\frac{s p}{p-1}}} \leq \frac{C \theta^{s p \frac{2-p}{p-1}}}{R^{s p \frac{2-p}{p-1}}|x|^{N+s p}} .
$$

We thus conclude that $\left(-\Delta_{p}\right)^{s} U \leq\left(-\Delta_{p}\right)^{s} w$ in ${\overline{B_{R / \theta}}}^{c}$ for $R$ sufficiently large, as desired.

\section{Appendix A. Power functions}

We have the following result on power functions.

Lemma A.1. Let $0<(N-s p) / p<\beta<N /(p-1)$. For every $R>0$, the function $x \mapsto|x|^{-\beta}$ belongs to $\widetilde{D}^{s, p}\left({\overline{B_{R}}}^{c}\right)$.

Proof. A direct computation shows that $x \mapsto|x|^{-\beta}$ belongs to $L_{\mathrm{loc}}^{p-1}\left(\mathbb{R}^{N}\right) \cap L^{p^{*}}\left(B_{R}^{c}\right)$, when $\beta$ is as in the statement. We take $r<R$, then $E={\overline{B_{r}}}^{c} \supset{\overline{B_{R}}}^{c}$ and we need to show

$$
\left[|x|^{-\beta}\right]_{W^{s, p}\left(B_{r}^{c}\right)}<+\infty, \quad \text { for } \frac{N-s p}{p}<\beta .
$$

We compute in polar coordinates

$$
\begin{aligned}
\int_{B_{r}^{c} \times B_{r}^{c}} & \frac{\left.|| x\right|^{-\beta}-\left.|y|^{-\beta}\right|^{p}}{|x-y|^{N+s p}} d x d y=\int_{\mathbf{S}^{N-1} \times \mathbf{S}^{N-1}} \int_{r}^{+\infty} \int_{r}^{+\infty} \frac{\left|\varrho^{-\beta}-t^{-\beta}\right|^{p} \varrho^{N-1} t^{N-1}}{\left|\varrho \omega_{1}-t \omega_{2}\right|^{N+s p}} d \varrho d t d \omega_{1} d \omega_{2} \\
& =2 \int_{r}^{+\infty} \frac{\varrho^{-\beta p} \varrho^{2 N-2}}{\varrho^{N+s p}} \int_{r}^{\varrho}\left|1-\left(\frac{t}{\varrho}\right)^{-\beta}\right|^{p} \int_{\mathbf{S}^{N-1} \times \mathbf{S}^{N-1}} \frac{d \omega_{1} d \omega_{2}}{\left|\omega_{1}-\left(t \omega_{2}\right) / \varrho\right|^{N+s p}}\left(\frac{t}{\varrho}\right)^{N-1} d t d \varrho \\
& =2 \int_{r}^{+\infty} \frac{\varrho^{-\beta p} \varrho^{2 N-1}}{\varrho^{N+s p}} \int_{r / \varrho}^{1}\left|1-\xi^{-\beta}\right|^{p} \xi^{N-1} \int_{\mathbf{S}^{N-1} \times \mathbf{S}^{N-1}} \frac{d \omega_{1} d \omega_{2}}{\left|\omega_{1}-\xi \omega_{2}\right|^{N+s p}} d \xi d \varrho .
\end{aligned}
$$

Let us now prove that for $0<\xi<1$ it holds

$$
\int_{\mathbf{S}^{N-1} \times \mathbf{S}^{N-1}} \frac{d \omega_{1} d \omega_{2}}{\left|\omega_{1}-\xi \omega_{2}\right|^{N+s p}} \leq \frac{C}{(1-\xi)^{1+s p}}
$$

Without loss of generality, we may assume that $\xi \geq 1 / 2$, since for $0<\xi<1 / 2$ the integral is uniformly bounded. By rotational invariance, we have

$$
\int_{\mathbf{S}^{N-1} \times \mathbf{S}^{N-1}} \frac{d \omega_{1} d \omega_{2}}{\left|\omega_{1}-\xi \omega_{2}\right|^{N+s p}}=\left|\mathbf{S}^{N-1}\right| \int_{\mathbf{S}^{N-1}} \frac{d \omega_{2}}{\left|\mathbf{e}_{1}-\xi \omega_{2}\right|^{N+s p}},
$$


where $\mathbf{e}_{1}=(1,0, \ldots, 0)$. By changing variable $\omega_{2}=(t, z)$ with

$$
t= \pm \sqrt{1-|z|^{2}}, \quad z \in B_{1}^{\prime} \subset \mathbb{R}^{N-1},
$$

we therefore get (the constant $C$ may vary from a line to another)

$$
\begin{aligned}
\int_{\mathbf{S}^{N-1}} \frac{d \omega_{2}}{\left|\mathbf{e}_{1}-\xi \omega_{2}\right|^{N+s p}} & =\int_{\mathbf{S}^{N-1} \backslash B_{1}\left(\mathbf{e}_{1}\right)} \frac{d \omega_{2}}{\left|\mathbf{e}_{1}-\xi \omega_{2}\right|^{N+s p}}+\int_{\mathbf{S}^{N-1} \cap B_{1}\left(\mathbf{e}_{1}\right)} \frac{d \omega_{2}}{\left|\mathbf{e}_{1}-\xi \omega_{2}\right|^{N+s p}} \\
& \leq C\left(1+\int_{B_{1}^{\prime}} \frac{d z}{\left((1-\xi t)^{2}+\xi^{2}|z|^{2}\right)^{\frac{N+s p}{2}}}\right) \\
& \leq C\left(1+\int_{B_{1}^{\prime}} \frac{d z}{\left((1-\xi)^{2}+\xi^{2}|z|^{2}\right)^{\frac{N+s p}{2}}}\right) \\
& \leq C\left(1+\frac{1}{(1-\xi)^{1+s p}} \int_{B^{\prime} \frac{\xi}{1-\xi}} \frac{1}{\left(1+|y|^{2}\right)^{\frac{N+s p}{2}}} d y\right) \\
& \leq C\left(1+\frac{1}{(1-\xi)^{1+s p}} \int_{\mathbb{R}^{N-1}} \frac{1}{\left(1+|y|^{2}\right)^{\frac{N+s p}{2}}} d y\right)
\end{aligned}
$$

which proves the claim. Taking into account that for $0<\xi<1$ it also holds

$$
\frac{\left|1-\xi^{-\beta}\right|^{p}}{|1-\xi|^{1+s p}} \leq C\left(\xi^{-\beta p}+|1-\xi|^{p(1-s)-1}\right)
$$

we therefore get

$$
\left[|x|^{-\beta}\right]_{W^{s, p}\left(B_{r}^{c}\right)}^{p} \leq C \int_{r}^{+\infty} \varrho^{N-1-p(s+\beta)} d \varrho \int_{r / \varrho}^{1} \xi^{N-1}\left(\xi^{-\beta p}+|1-\xi|^{p(1-s)-1}\right) d \xi .
$$

All the integrals are now explicitly computable and one can readily get (A.1).

Lemma A.2. Let $0<(N-s p) / p<\beta<N /(p-1)$. For every $R>0$, it holds

$$
\left(-\Delta_{p}\right)^{s}|x|^{-\beta}=C(\beta)|x|^{-\beta(p-1)-s p} \quad \text { weakly in }{\overline{B_{R}}}^{c} \text {, }
$$

where the constant $C(\beta)$ is given by

$$
C(\beta)=2 \int_{0}^{1} \varrho^{s p-1}\left[1-\varrho^{N-s p-\beta(p-1)}\right]\left|1-\varrho^{\beta}\right|^{p-1} \Phi(\varrho) d \varrho,
$$

and

$$
\Phi(\varrho)=\mathcal{H}^{N-2}\left(\mathbf{S}^{N-2}\right) \int_{-1}^{1} \frac{\left(1-t^{2}\right)^{\frac{N-3}{2}}}{\left(1-2 t \varrho+\varrho^{2}\right)^{\frac{N+s p}{2}}} d t .
$$

Proof. Observe that

$$
|x|^{-\beta(p-1)-s p} \in L^{(p *)^{\prime}}\left(B_{R}^{c}\right), \quad \text { for any } \beta>(N-s p) / p .
$$

Then, by Theorem 2.1 and Proposition 2.5 it suffices to show that

$$
\int_{\mathbb{R}^{2 N}} \frac{J_{p}\left(|x|^{-\beta}-|y|^{-\beta}\right)}{|x-y|^{N+s p}}(\varphi(x)-\varphi(y)) d x d y=C(\beta) \int_{\Omega}|x|^{-\beta(p-1)-s p} \varphi d x,
$$


for an arbitrary $\varphi \in C_{c}^{\infty}\left({\overline{B_{R}}}^{c}\right)$. For every such a $\varphi$ we consider the double integral

$$
\int_{\mathbb{R}^{2 N}} \frac{J_{p}\left(|x|^{-\beta}-|y|^{-\beta}\right)}{|x-y|^{N+s p}}(\varphi(x)-\varphi(y)) d x d y .
$$

We observe that this is absolutely convergent, indeed

$$
\begin{aligned}
\int_{\mathbb{R}^{2 N}} & \frac{\left|J_{p}\left(|x|^{-\beta}-|y|^{-\beta}\right)\right|}{|x-y|^{N+s p}}|\varphi(x)-\varphi(y)| d x d y \\
\quad & \int_{B_{R}^{c} \times B_{R}^{c}} \frac{\left|J_{p}\left(|x|^{-\beta}-|y|^{-\beta}\right)\right|}{|x-y|^{N+s p}}|\varphi(x)-\varphi(y)| d x d y \\
& +2 \int_{B_{R}} \int_{\operatorname{supp}(\varphi)} \frac{\left|J_{p}\left(|x|^{-\beta}-|y|^{-\beta}\right)\right|}{|x-y|^{N+s p}}|\varphi(y)| d x d y \\
\leq & {\left[|x|^{-\beta}\right]_{W^{s, p}\left(B_{R}^{c}\right)}[\varphi]_{W^{s, p}\left(B_{R}^{c}\right)}+C\|\varphi\|_{L^{\infty}}|\operatorname{supp}(\varphi)| \int_{B_{R}}|x|^{-\beta(p-1)} d x }
\end{aligned}
$$

and both terms are finite, thanks to Lemma A.1. For $\delta>0$ we consider the conical set

$$
\mathcal{O}_{\delta}=\left\{(x, y) \in \mathbb{R}^{2 N}:(1-\delta)|x| \leq|y| \leq(1+\delta)|x|\right\},
$$

then by the Dominated Convergence Theorem

$$
\begin{aligned}
\lim _{\delta \searrow 0} \int_{\mathcal{O}_{\delta}^{c}} \frac{J_{p}\left(|x|^{-\beta}-|y|^{-\beta}\right)}{|x-y|^{N+s p}}(\varphi(x)-\varphi(y)) d y d x \\
\quad=\int_{\mathbb{R}^{2 N}} \frac{J_{p}\left(|x|^{-\beta}-|y|^{-\beta}\right)}{|x-y|^{N+s p}}(\varphi(x)-\varphi(y)) d x d y
\end{aligned}
$$

We now observe that

$$
\int_{\mathcal{O}_{\delta}^{c}} \frac{J_{p}\left(|x|^{-\beta}-|y|^{-\beta}\right)}{|x-y|^{N+s p}}(\varphi(x)-\varphi(y)) d y d x=2 \int_{\mathbb{R}^{N}}\left(\int_{\mathcal{K}_{\delta}(x)^{c}} \frac{J_{p}\left(|x|^{-\beta}-|y|^{-\beta}\right)}{|x-y|^{N+s p}} d y\right) \varphi(x) d x
$$

where for every $x \in \mathbb{R}^{N}$

$$
\mathcal{K}_{\delta}(x)=\left\{y \in \mathbb{R}^{N}:(1-\delta)|x| \leq|y| \leq(1+\delta)|x|\right\},
$$

and of course $\mathcal{K}_{\delta}(x)=\mathcal{K}_{\delta}\left(x^{\prime}\right)$ whenever $|x|=\left|x^{\prime}\right|$. We set

$$
f_{\delta}(x)=2 \int_{\mathcal{K}_{\delta}(x)^{c}} \frac{J_{p}\left(|x|^{-\beta}-|y|^{-\beta}\right)}{|x-y|^{N+s p}} d y, \quad x \in \mathbb{R}^{N} \backslash\{0\},
$$

it is easily seen that $f_{\delta}$ is a radial function, homogeneous of degree $-\beta(p-1)-s p$ (see [4, Lemma 6.2]). Thus for $x \neq 0$ we have

$$
f_{\delta}(x)=|x|^{-\beta(p-1)-s p} f_{\delta}(\omega), \quad \text { for } \quad \omega=\frac{x}{|x|} \in \mathbf{S}^{N-1}
$$

We set

$$
C(\beta ; \delta):=f_{\delta}(\omega)=2 \int_{\mathcal{K}_{\delta}(\omega)^{c}} \frac{J_{p}\left(1-|y|^{-\beta}\right)}{|\omega-y|^{N+s p}} d y, \quad \omega \in \mathbf{S}^{N-1}
$$


which is independent of the direction $\omega$, by radiality of $f_{\delta}$. By taking the average over $\mathbf{S}^{N-1}$ and proceeding as in [4, Lemma B.2], we get

$$
C(\beta ; \delta)=2 \int_{|\varrho-1| \geq \delta} \varrho^{N-1}\left|1-\varrho^{-\beta}\right|^{p-2}\left(1-\varrho^{-\beta}\right) \Phi(\varrho) d \varrho
$$

where $\Phi$ is defined in (A.3). We now decompose the integral defining $C(\beta ; \delta)$ and perform a change of variables, i.e.

$$
\begin{aligned}
C(\beta ; \delta)= & -2 \int_{0}^{1-\delta} \varrho^{N-1}\left|1-\varrho^{-\beta}\right|^{p-1} \Phi(\varrho) d \varrho+2 \int_{1+\delta}^{\infty} \varrho^{N-1}\left|1-\varrho^{-\beta}\right|^{p-1} \Phi(\varrho) d \varrho \\
= & -2 \int_{0}^{1-\delta} \varrho^{N-1-\beta(p-1)}\left|\varrho^{\beta}-1\right|^{p-1} \Phi(\varrho) d \varrho \\
& +2 \int_{0}^{1 /(1+\delta)} \varrho^{-N-1}\left|1-\varrho^{\beta}\right|^{p-1} \Phi(1 / \varrho) d \varrho .
\end{aligned}
$$

Finally, observe that

$$
\Phi(1 / \varrho)=\varrho^{N+s p} \Phi(\varrho)
$$

thus the quantity $C(\beta ; \delta)$ can be written as

$$
\begin{aligned}
C(\beta ; \delta)= & 2 \int_{0}^{1-\delta}\left(1-\varrho^{N-s p-\beta(p-1)}\right) \varrho^{s p-1}\left(1-\varrho^{\beta}\right)^{p-1} \Phi(\varrho) d \varrho \\
& +2 \int_{1-\delta}^{1 /(1+\delta)} \varrho^{s p-1}\left(1-\varrho^{\beta}\right)^{p-1} \Phi(\varrho) d \varrho .
\end{aligned}
$$

Recall that $\varphi$ is compactly supported in ${\overline{B_{R}}}^{c}$, thus by using (A.4) we can estimate

$$
\left.\left|\int_{\Omega} f_{\delta} \varphi d x-C(\beta) \int_{\Omega}\right| x\right|^{-\beta(p-1)-s p} \varphi d x\left|\leq\|\varphi\|_{\infty} R^{-\beta(p-1)-s p}\right| \operatorname{supp}(\varphi)|| C(\beta ; \delta)-C(\beta) \mid .
$$

In order to prove that $C(\beta ; \delta)$ converges to $C(\beta)$ as $\delta$ goes to 0 , we decompose the function $\Phi$ defined in (A.3) as follows

$$
\Phi(\varrho)=\int_{-1}^{1 / 2} \frac{\left(1-t^{2}\right)^{\frac{N-3}{2}}}{\left(1-2 t \varrho+\varrho^{2}\right)^{\frac{N+s p}{2}}} d t+\int_{1 / 2}^{1} \frac{\left(1-t^{2}\right)^{\frac{N-3}{2}}}{\left(1-2 t \varrho+\varrho^{2}\right)^{\frac{N+s p}{2}}} d t=: \Phi_{1}(\varrho)+\Phi_{2}(\varrho),
$$

where we omitted the dimensional constant $\mathcal{H}^{N-2}\left(\mathbf{S}^{N-2}\right)$ for simplicity. Let us start estimating $\Phi_{1}$. If we use that

we get

$$
1-2 t \varrho+\varrho^{2}=(\varrho-t)^{2}+\left(1-t^{2}\right) \geq \frac{3}{4}, \quad \text { if }-1 \leq t \leq \frac{1}{2},
$$

$$
0 \leq \Phi_{1}(\varrho) \leq C, \quad 0<\varrho<1 .
$$

We now consider $\Phi_{2}(\varrho)$, discussing separately the cases $0<\varrho<1 / 2$ and $1 / 2 \leq \varrho<1$. We observe that for $0<\varrho<1 / 2$ we have

$$
1-2 t \varrho+\varrho^{2}=(1-\varrho)^{2}+2 \varrho(1-t) \geq \frac{1}{4}, \quad \text { if } \frac{1}{2} \leq t \leq 1 .
$$

Then we get again

$$
0 \leq \Phi_{2}(\varrho) \leq C, \quad \text { if } 0<\varrho<\frac{1}{2}
$$


We are left with the term $\Phi_{2}(\varrho)$ for $1 / 2 \leq \varrho<1$. With simple manipulations ${ }^{4}$ we can write it as

$$
\Phi_{2}(\varrho)=\frac{(2 \varrho)^{-\frac{N-1}{2}}}{(1-\varrho)^{1+s p}} \int_{0}^{\frac{\varrho}{(1-\varrho)^{2}}} \frac{\left(2-\frac{(1-\varrho)^{2}}{2 \varrho} \tau\right)^{\frac{N-3}{2}} \tau^{\frac{N-3}{2}}}{(1+\tau)^{\frac{N+s p}{2}}} d \tau .
$$

In particular, we get

$$
0 \leq \Phi_{2}(\varrho) \leq C(1-\varrho)^{-1-s p}, \quad \text { if } \frac{1}{2} \leq \varrho<1 .
$$

By using (A.6), (A.7) and (A.8), we thus obtain for the first integral in (A.5)

$$
\lim _{\delta \searrow 0} 2 \int_{0}^{1-\delta}\left(1-\varrho^{N-s p-\beta(p-1)}\right) \varrho^{s p-1}\left(1-\varrho^{\beta}\right)^{p-1} \Phi(\varrho) d \varrho=C(\beta),
$$

and observe that the latter is finite, thanks to (A.8). It is only left to show that the other integral in (A.5) converges to 0. Still by (A.6) and (A.8), we obtain

$$
\begin{aligned}
\lim _{\delta \searrow 0} \int_{1-\delta}^{1 /(1+\delta)} & \varrho^{s p-1}\left(1-\varrho^{\beta}\right)^{p-1} \Phi(\varrho) d \varrho \\
& \leq C \lim _{\delta \searrow 0} \int_{1-\delta}^{1 /(1+\delta)} \varrho^{s p-1}\left(1-\varrho^{\beta}\right)^{p-1} d \varrho \\
& +C \lim _{\delta \searrow 0} \int_{1-\delta}^{1 /(1+\delta)} \varrho^{s p-1}\left(1-\varrho^{\beta}\right)^{p-1}(1-\varrho)^{-1-s p} d \varrho \\
& \leq C \lim _{\delta \searrow 0} \int_{1-\delta}^{1 /(1+\delta)}(1-\varrho)^{p-2-s p} d \varrho \\
& =\frac{C}{p-1-s p} \lim _{\delta \searrow 0}\left[-\left(\frac{\delta}{1+\delta}\right)^{p-1-s p}+\delta^{p-1-s p}\right],
\end{aligned}
$$

where we assumed for simplicity that $p-1-s p \neq 0$. If $p-1-s p>0$, the last term converges to 0 . If $p-1-s p<0$, we have

$$
\begin{aligned}
\left(\frac{\delta}{1+\delta}\right)^{p-1-s p}-\delta^{p-1-s p} & =\delta^{p-1-s p}\left[(1+\delta)^{s p+1-p}-1\right] \\
& \simeq(s p+1-p) \delta^{p-s p}, \quad \text { as } \delta \searrow 0,
\end{aligned}
$$

and thus the integral converges to 0 again. Finally, the borderline case $p-1-s p=0$ is treated similarly, we leave the details to the reader.

In conclusion, we get

$$
\lim _{\delta \searrow 0} \int_{\Omega} f_{\delta} \varphi d x=C(\beta) \int_{\Omega}|x|^{-\beta(p-1)-s p} \varphi d x,
$$

as desired.

Remark A.3. The previous result was proved in [15, Lemma 3.1] for the limit case $\beta=$ $(N-s p) / p$. Our argument is different, since we rely on elementary estimates for the function $\Phi$, rather than on special properties of hypergeometric and beta functions like in [15].

\footnotetext{
${ }^{4}$ We use the change of variables $\tau=\frac{2 \varrho}{(1-\varrho)^{2}}(1-t)$.
} 
Observe that the choice $\beta=(N-s p) /(p-1)$ is feasible in the previous results, since

$$
\frac{N-s p}{p}<\frac{N-s p}{p-1}<\frac{N}{p-1}
$$

Moreover, with such a choice we have $C(\beta)=0$ in (A.2). Then from Lemmas A.1 and A.2, we get the following.

Theorem A.4. For any $R>0, \Gamma(x)=|x|^{-\frac{N-s p}{p-1}}$ belongs to $\widetilde{D}^{s, p}\left({\overline{B_{R}}}^{c}\right)$ and weakly solves $\left(-\Delta_{p}\right)^{s} u=0$ in ${\overline{B_{R}}}^{c}$.

\section{REFERENCES}

[1] F. J. Almgren, E. Lieb, Symmetric decreasing rearrangement is sometimes continuous, J. Amer. Math. Soc., 2 (1989), 683-773. 12

[2] F. Andreu, J. M. Mazón, J. D. Rossi, J. Toledo, A nonlocal $p$-Laplacian evolution equation with nonhomogeneous Dirichlet boundary conditions, SIAM J. Math. Anal., 40 (2009), 1815-1851. 2

[3] T. Aubin, Problèmes isopérimétriques et espaces de Sobolev, J. Differential Geometry, 11 (1976), 573598. 1

[4] L. Brasco, G. Franzina, Convexity properties of Dirichlet integrals and Picone-type inequalities, Kodai Math. J., 37 (2014), 769-799. 27, 28

[5] L. Brasco, E. PArini, The second eigenvalue of the fractional $p$-Laplacian, Adv. Calc. Var., to appear. $12,13,15$

[6] L. Brasco, E. Lindgren, E. Parini, The fractional Cheeger problem, Interfaces Free Bound., 16 (2014), 419-458. 2

[7] W. Chen, C. Li, B. Ou, Classification of solutions for an integral equation, Comm. Pure Appl. Math., 59 (2006), 330-343. 3

[8] D. Cordero-Erausquin, B. Nazaret, C. Villani, A mass-transportation approach to sharp Sobolev and Gagliardo-Nirenberg inequalities, Adv. Math., 182 (2004), 307-332. 2

[9] A. Cotsiolis, N. Tavoularis, Best constants for Sobolev inequalities for higher order fractional derivatives, J. Math. Anal. Appl., 295 (2004), 225-236. 3

[10] L. Damascelli, S. Merchán, L. Montoro, B. Sciunzi, Radial symmetry and applications for a problem involving the $-\Delta_{p}(\cdot)$ operator and critical nonlinerity in $\mathbb{R}^{N}$, Adv. Math., 265 (2014), 313-335. 2

[11] A. Di Castro, T. Kuusi, G. Palatucci, Nonlocal Harnack inequalities, J. Funct. Anal., 267 (2014), 1807-1836. 2

[12] E. Di Nezza, G. Palatucci, E. Valdinoci, Hitchhiker's guide to the fractional Sobolev spaces, Bull. Sci. Math., 136 (2012), 521-573. 3, 7

[13] A. Fiscella, R. Servadei, E. Valdinoci, Density properties for fractional Sobolev spaces, Ann. Acad. Sci. Fenn. Math., 40 (2015), 235-253. 6

[14] H. Federer, W. H. Fleming, Normal and integral currents, Ann. Math., 72 (1960) 458-520. 1

[15] R. L. Frank, R. Seiringer, Non-linear ground state representations and sharp Hardy inequalities, J. Funct. Anal., 255 (2008), 3407-3430. 2, 6, 12, 29

[16] N. Gigli, S. Mosconi, The abstract Lewy-Stampacchia inequality and applications, J. Math. Pures Appl., 104 (2015), 258-275. 17

[17] M. Guedda, L. Veron, Local and global properties of solutions of quasilinear elliptic equations, J. Differential Equations, 76 (1988), 159-189. 2, 4

[18] A. Iannizzotto, S. Liu, K. Perera, M. Squassina, Existence results for fractional $p$-Laplacian problems via Morse theory, Adv. Calc. Var. (2015), to appear. 2

[19] A. Innnizzotto, S. Mosconi, M. Squassina, Global Hölder regularity for the fractional $p$-Laplacian, to appear on Rev. Mat. Iberoamericana, available at http://arxiv.org/abs/1411:2956 2, 4, 5, 8, 9, 12, 17,19 
[20] A. Innnizzotto, S. Mosconi, M. Squassina, A note on global regularity for the weak solutions of fractional $p$-Laplacian equations, to appear on Atti Accad. Naz. Lincei Rend. Lincei Mat. Appl., available at http://arxiv.org/abs/1504.01006 2

[21] H. Ishit, G. Nakamura, A class of integral equations and approximation of $p$-Laplace equations, Calc. Var. Partial Differential Equations, 37 (2010), 485-522. 2

[22] T. Kuusi, G. Mingione, Y. Sire, Nonlocal equations with measure data, Comm. Math. Phys., 337 (2015), 1317-1368. 2

[23] E. H. Lieb, Sharp constants in the Hardy-Littlewood-Sobolev and related inequalities, Ann. Math., 118 (1983), 349-374. 3

[24] E. H. Lieb, M. Loss, Analysis, Second edition. Graduate Studies in Mathematics, 14. American Mathematical Society, Providence, RI, 2001. 10

[25] P. L. Lions, The concentration-compactness principle in the calculus of variations. The limit case. I, Rev. Mat. Iberoamericana, 1 (1985), 145-201. 12

[26] E. Lindgren, P. Lindqvist, Fractional eigenvalues, Calc. Var. Partial Differential Equations, 49 (2014), 795-826. 9

[27] V. G. Maz'JA, Classes of regions and imbedding theorems for function spaces, Soviet Math. Dokl., 1 (1960), 882-885. 1

[28] V. Maz'ya, T. Shaposhnikova, On the Bourgain, Brezis, and Mironescu theorem concerning limiting embeddings of fractional Sobolev spaces, J. Funct. Anal., 195 (2002), 230-238. 2

[29] S. Mosconi, K. Perera, M. Squassina, Y. Yang, On the Brezis-Nirenberg problem for the fractional p-Laplacian, preprint (2015), available at http://arxiv.org/abs/1508.00700 4

[30] G. Rosen, Minimal value for $c$ in the Sobolev inequality, SIAM J. Appl. Math., 21 (1971), 30-33. 2

[31] R. Servadei, E. Valdinoci, The Brezis-Nirenberg result for the fractional Laplacian, Trans. Amer. Math. Soc., 367 (2015), 67-102. 4

[32] B. ScIunzI, Classification of positive $\mathcal{D}^{1, p}\left(\mathbb{R}^{N}\right)$-solutions to the critical $p$-Laplace equation in $\mathbb{R}^{N}$, preprint (2015), available at http://arxiv.org/abs/1506.036532

[33] G. Thlenti, Best constant in Sobolev inequality, Ann. Mat. Pura Appl., 110 (1976), 353-372. 1

[34] J. Vetois, A priori estimates and application to the symmetry of solutions for critical $p$-Laplace equations, to appear on J. Differential Equations (2015), doi:10.1016/j.jde.2015.08.0412

(L. Brasco) Aix-Marseille Université, CNRS

Centrale Marseille, I2M, UMR 7373, 39 Rue Frédéric Joliot Curie

13453 Marseille, France

E-mail address: lorenzo.brasco@univ-amu.fr

(S. Mosconi \& M. Squassina) Dipartimento Di Informatica

Università degli Studi di Verona

VERONA, ITALY

E-mail address: sunrajohannes.mosconi@univr.it

E-mail address: marco.squassina@univr.it 